\title{
Nernst-Planck Based Description of Transport, Coulombic Interactions and Geochemical Reactions in Porous Media: Modeling Approach and Benchmark Experiments
}

Rolle, Massimo; Sprocati, Riccardo; Masi, Matteo; Jin, Biao; Muniruzzaman, Muhammad

Published in:

Water Resources Research

Link to article, DOI:

10.1002/2017WR022344

Publication date:

2018

Document Version

Peer reviewed version

Link back to DTU Orbit

Citation (APA):

Rolle, M., Sprocati, R., Masi, M., Jin, B., \& Muniruzzaman, M. (2018). Nernst-Planck Based Description of Transport, Coulombic Interactions and Geochemical Reactions in Porous Media: Modeling Approach and Benchmark Experiments. Water Resources Research, 54(4), 3176-3195.

https://doi.org/10.1002/2017WR022344

\section{General rights}

Copyright and moral rights for the publications made accessible in the public portal are retained by the authors and/or other copyright owners and it is a condition of accessing publications that users recognise and abide by the legal requirements associated with these rights.

- Users may download and print one copy of any publication from the public portal for the purpose of private study or research.

- You may not further distribute the material or use it for any profit-making activity or commercial gain

- You may freely distribute the URL identifying the publication in the public portal 


\title{
Nernst-Planck based description of transport, Coulombic interactions and geochemical reactions in porous media: Modeling approach and benchmark experiments
}

\author{
Massimo Rolle ${ }^{1}$ (D) Riccardo Sprocati $^{1}$, Matteo Masi ${ }^{2}$, Biao Jin ${ }^{3}$, and Muhammad \\ Muniruzzaman ${ }^{4}$
}

${ }^{1}$ Department of Environmental Engineering, Technical University of Denmark, Bygningstorvet, Building 115, 2800 Kgs. Lyngby, Denmark

${ }^{2}$ Department of Energy, Systems, Territory, and Construction Engineering, University of Pisa, Via C.F. Gabba 22, 56122 Pisa, Italy

${ }^{3}$ State Key Laboratory of Organic Geochemistry, Guangzhou Institute of Geochemistry, Chinese Academy of Sciences, Kehua Street 511, 510640 Guangzhou, China

${ }^{4}$ Geological Survey of Finland, Neulaniementie 5, 70211 Kuopio, Finland

Corresponding author: Massimo Rolle (masro@env.dtu.dk)

\section{Key Points:}

- Nernst-Planck based approach to model multidimensional transport, charge interactions and chemical reactions in flow-through porous media

- Computation and visualization of diffusive/dispersive, electromigration and activity coefficients' gradient fluxes

- Code validated with high-resolution 2-D experimental data and first fully 3-D dataset on multicomponent ionic transport

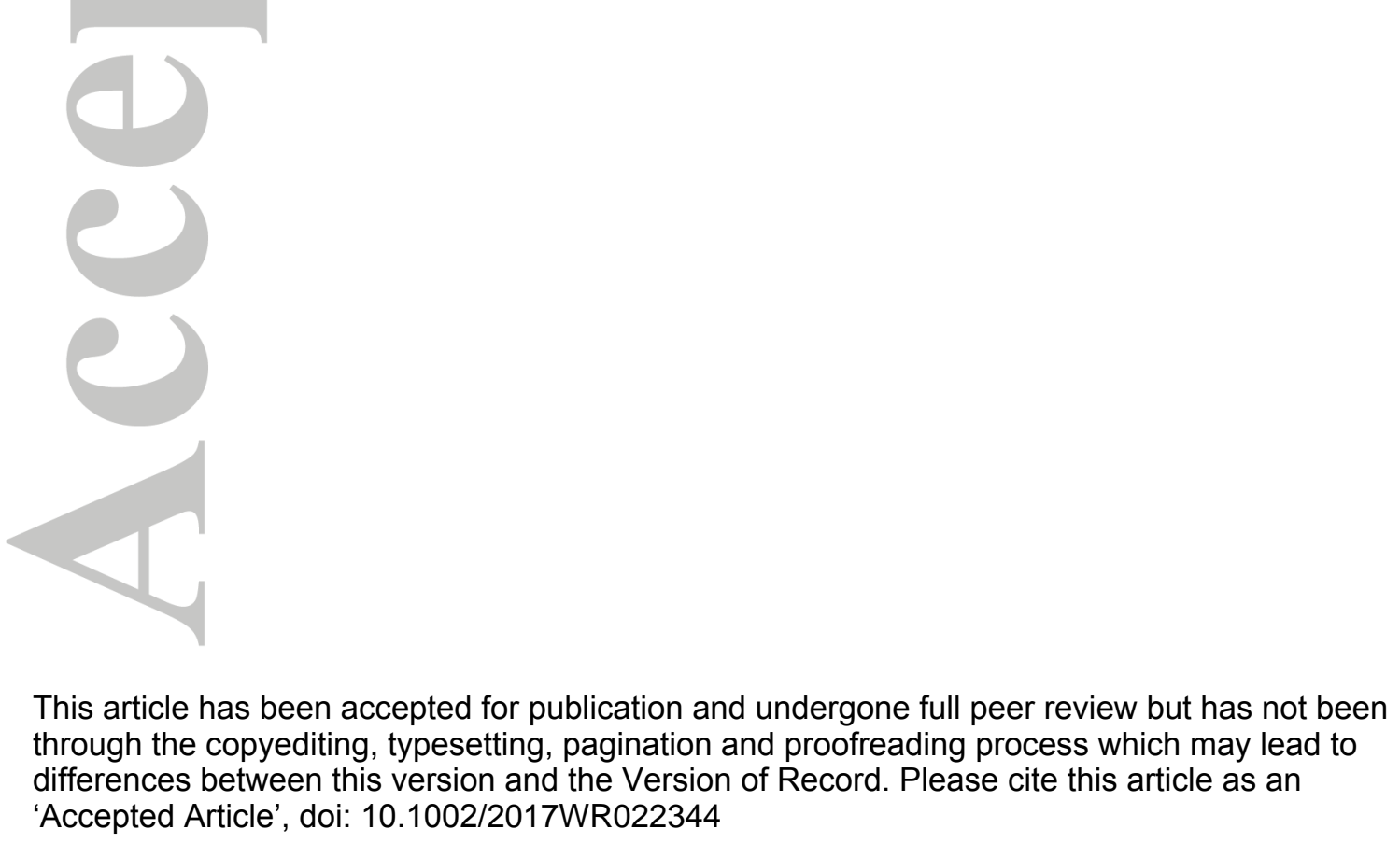




\section{Abstract}

Transport of multicomponent electrolyte solutions in saturated porous media is affected by the electrostatic interactions between charged species. Such Coulombic interactions couple the displacement of the different ions in the pore water and remarkably impact mass transfer not only under diffusion- but also under advection-dominated flow regimes. To accurately describe charge effects in flow-through systems, we propose a multidimensional modeling approach based on the Nernst-Planck formulation of diffusive/dispersive fluxes. The approach is implemented with a COMSOL-PhreeqcRM coupling allowing us to solve multicomponent ionic conservative and reactive transport problems, in domains with different dimensionality (1-D, 2-D and 3-D), and in homogeneous and heterogeneous media. The Nernst-Planck based coupling has been benchmarked with analytical solutions, numerical simulations with another code, and highresolution experimental datasets. The latter include flow-through experiments that have been carried out in this study to explore the effects of electrostatic interactions in fully threedimensional setups. The results of the simulations show excellent agreement for all the benchmarks problems, which were selected to illustrate the capabilities and the distinct features of the Nernst-Planck based reactive transport code. The outcomes of this study illustrate the importance of Coulombic interactions during conservative and reactive transport of charged species in porous media and allow the quantification and visualization of the specific contributions to the diffusive/dispersive Nernst-Planck fluxes, including the Fickian component, the term arising from the activity coefficient gradients, and the contribution due to electromigration.

\section{Introduction}

Solute transport in porous media depends on the interplay of physical, chemical, electrochemical and biological processes. In particular, the diffusive movement of charged species in solution, such as major ions and many contaminants in groundwater systems, results from the gradient in electrochemical potential (Bard \& Faulkner, 2001). Thus, Nernst-Planck equations for fluxes and conservation laws describe the movement of the charged species (Probenstein, 1989). A common assumption in groundwater applications is to neglect the electrostatic interactions between charged species in pore water and to describe solute displacement by Fickian diffusion. However, a number of contributions, mainly from the geochemistry literature, have focused on the importance of multicomponent ionic transport (BenYakov, 1972; Lasaga, 1979; Gvirtzman \& Gorelick, 1991; Boudreau et al., 2004; Steefel and Maher, 2009) and have shown the need of taking into account Coulombic interactions in practical applications in diffusion-dominated low-permeability matrices (Giambalvo et al., 2002; Appelo \& Wersin, 2007; Liu, 2007; Appelo et al., 2008; Liu et al., 2011; Tournassat \& Appelo, 2011; Alt-Epping et al., 2015; Tournassat \& Steefel, 2015). A few recent experimental studies (e.g., Rolle et al., 2013a, Muniruzzaman et al., 2014; Muniruzzaman \& Rolle, 2017) have shown the effects of electrostatic interactions between charged species during transport of strong electrolytes in porous media under advection-dominated flow-through conditions. Besides these experimental evidences for electrolyte transport, a number of pore-scale studies showed the importance of diffusion and incomplete mixing in pore throats and channels also at high flow velocities and Péclet numbers. Pore-scale investigations were based on both microfluidic experiments (Li et al., 2008; Zhang et al., 2010; de Anna et al., 2014; Scheven et al., 2013) and pore-scale simulations (e.g., Cao \& Kitanidis, 1998; Tartakovsky et al., 2009; Molins et al., 2012; Rolle et al., 2012; Yoon et al., 2012; Rolle \& Kitanidis, 2014; Crevacore et al., 2016). In 
addition to the results of laboratory experiments, the outcomes of such pore-scale investigations have also been instrumental to improve the parameterization of key macroscopic quantities, such as hydrodynamic dispersion coefficients (e.g., Delgado, 2006; Bijelijc \& Blunt, 2007; Chiogna et al., 2010; Rolle et al., 2012), necessary for the continuum-based description of solute transport in groundwater studies. The effects of small scale diffusive processes propagate through the scales and have been shown to impact macroscopic solute transport at field scales both for conservative solutes (e.g., Carrera et al., 1998; LaBolle \& Fogg, 2001; Chiogna et al., 2011; Rolle et al., 2013b; Hadley \& Newell, 2014) as well as for mixing-controlled reactive transport (e.g., Chiogna et al., 2011; Cirpka et al., 2012; Eckert et al., 2012). Despite the controlling role of small scale diffusive processes for groundwater transport is increasingly recognized, the macroscopic impact of electrostatic interactions on transport of charged species in flow-through porous media has been hardly explored. Possible reasons of this knowledge gap might be the difficulties in performing high-resolution experiments allowing resolving the effects of electromigration under flow-through conditions and without forcing an electric field, as well as the scarcity of simulation tools allowing multi-dimensional reactive transport modeling considering multicomponent ionic transport (Steefel et al., 2015; Rasouli et al., 2015; Muniruzzaman \& Rolle, 2016). Exploring electrostatic interactions under flow-through conditions is of pivotal importance to improve our capability to understand and predict the behavior of complex geochemical systems. Transport and mixing of major ions and charged contaminants, including acidic plumes and a number of trace metals and metalloids of primary environmental concern (Kjøller et al., 2004; Prigiobbe et al., 2012; Rolle et al. 2013; Prigiobbe \& Bryant, 2014; McNeece \& Hesse 2017) strongly depend on electrostatic interactions with charged surfaces and within the pore water.

In this work we propose a solute transport formulation based on the Nernst-Planck equations to describe transport of charged species in saturated porous media. We present a modeling approach taking into account the Coulombic interactions between ions in the pore water solution and capable of performing a wide spectrum of reactive processes. The model is based on the coupling between the multiphysics simulator COMSOL Multiphysics ${ }^{\circledR}$ and the widely used geochemical code PHREEQC (Parkhurst \& Appelo, 2013). Such Nernst-Planck based, multicomponent ionic transport simulator is benchmarked and validated with analytical and numerical simulations, as well as with high-resolution data from flow-through experiments performed under advection-dominated flow conditions in quasi 2-D and in 3-D setups. The latter represent first experimental evidence of Coulombic effects during transport of strong electrolytes in fully three-dimensional porous media.

\section{Methods}

2.1 Modeling Approach

\subsubsection{Nernst-Planck equations in flow-through systems}

The diffusive displacement of dissolved ions in aqueous solution depends on the electrochemical potential $\mu_{i}$ of the ionic species $i$ :

$$
\mu_{i}=\mu_{i}^{0}+R T \ln a_{i}+z_{i} F \Phi
$$


where $\mu_{i}^{0}$ is the electrochemical potential in standard conditions, $R$ is the ideal gas constant, $T$ is the absolute temperature, $a_{i}$ is the activity of the ion in solution, $z_{\mathrm{i}}$ is the charge number, $F$ is the Faraday's constant and $\Phi$ is the electrostatic potential. Considering the spatial gradient of the electrochemical potential, the diffusive flux of the species $i$ is expressed by the Nernst-Planck equation:

$$
J_{i}=-D_{i}^{a q} \nabla C_{i}-D_{i}^{a q} C_{i} \nabla \ln \gamma_{i}-D_{i}^{a q} \frac{z_{i} F}{R T} C_{i} \nabla \Phi
$$

where $D_{i}^{a q}$ is the self-diffusion coefficient of the charged species $i$ in the aqueous solution, $C_{i}$ is the concentration and $\gamma_{i}$ is the activity coefficient. The first two terms on the righthand side of Eq. 2 describe the effect of the chemical potential gradient that result in the classic Fickian diffusion as well as in a contribution due to the gradient of the activity coefficients. The last term in Eq. 2 is the electromigration contribution and describes the effect of the electrostatic potential gradient arising from different transport velocities of the ions in the solution. Eq. 2 represents the corner stone to derive the multicomponent ionic diffusion equation of charged species in aqueous solutions.

In porous media diffusion occurs in the pore water and, under flow-through conditions, hydrodynamic dispersion affects solute displacement. As highlighted in both pore scale modeling (Tartakovsky et al., 2009; Hochstetler et al., 2013) and laboratory flow-through experiments in packed beds (Delgado \& Carvalho, 2001; Chiogna et al., 2010; Ye et al., 2015a) aqueous diffusion represents a quantitatively relevant contribution to hydrodynamic dispersion, especially in the transverse direction, not only at low but also at high flow velocities. Therefore, for transport of charged species in flow-through systems, a Nernst-Planck equation formally identical to Eq. 2 but considering the coefficients of hydrodynamic dispersion $\left(D_{i}\right)$, should be used:

$$
J_{i}=-D_{i} \nabla C_{i}-D_{i} C_{i} \nabla \ln \gamma_{i}-D_{i} \frac{z_{i} F}{R T} C_{i} \nabla \Phi
$$

The governing equation for multicomponent ionic transport in saturated porous media is derived from a mass balance over a control volume and integrating the fluxes due to advection and multicomponent ionic dispersion. The Nernst-Planck based equation for reactive transport of charged species in a multidimensional domain reads as:

$$
\frac{\partial C_{i}}{\partial t}+\boldsymbol{v} \cdot \nabla C_{i}-\nabla \cdot\left(\boldsymbol{J}_{i}\right)=r_{i}
$$

where $t$ is time, $v$ is the seepage velocity vector, $J_{i}$ is the total multicomponent ionic dispersion flux and $r_{i}$ is the reactive source/sink term.

\subsubsection{COMSOL-PhreeqcRM coupling for multicomponent ionic transport}

Coupling solute transport models with geochemical simulators has contributed to major advances in our capability to understand and quantitatively describe subsurface systems (Barry et al., 2002; Thullner et al., 2005; Steefel et al., 2015; Li et al., 2017). Many modern codes have extensive capabilities and have been used to describe reactive transport in different applications of environmental modeling of subsurface systems. In particular, for problems of solute transport in saturated porous media, examples of established reactive transport simulators including 
comprehensive features to describe geochemical and biogeochemical processes include CrunchFlow (Steefel \& Lasaga, 1994), Geochemist's Workbench (Bethke, 1997), MIN3P (e.g., Mayer et al., 2002; Molins et al., 2010), PHT3D (e.g., Prommer et al., 2003; Post \& Prommer, 2007; Appelo \& Rolle, 2010), TOUGHREACT (Xu \& Pruess, 2001) and PHAST (Parkhurst et al., 2004). In recent years, significant advances in the development of reactive transport codes have been favored by the increase of flexibility in coupling transport simulators with geochemical reactive codes. Notable examples are the modules IPhreeqc (Charlton \& Parkhurst, 2011) and PhreeqcRM (Parkhurst \& Wissmeier, 2015) that have been released to favor the interface of the widely used geochemical package PHREEQC (Parkhurst \& Appelo 2013) with other simulators (e.g., Kolditz et al., 2012; He et al., 2015; Korrani et al., 2015; Muniruzzaman \& Rolle, 2016). Different initiatives have proposed to adopt the IPhreeqc and PhreeqcRM modules to use the PHREEQC capabilities as reaction engine for the multi-purpose finite element code COMSOL Multiphysics ${ }^{\circledR}$ (e.g., Wissmeier \& Barry, 2011; Nardi et al., 2014; Masi et al., 2017; Jara et al., 2017).

Most of the simulators mentioned above are based on a Fickian description of diffusive/dispersive fluxes and only a few of them allow taking into account electromigration during transport of charged species (Steefel et al. 2015; Rasouli et al., 2015). In this work we develop a COMSOL-PhreeqcRM coupling, based on a Nernst-Planck formulation of conservative and reactive transport (Eqs. 1-4), allowing the description of physical displacement, Coulombic interactions and a wide range of geochemical reactions in multidimensional saturated porous media. The model is called NP-Phreeqc (Nernst-Planck-Phreeqc) and the coupling is performed with the LiveLink ${ }^{\mathrm{TM}}$ for MATLAB ${ }^{\circledR}$, a module developed by COMSOL Multiphysics ${ }^{\circledR}$ for communication with other software. The main steps of the proposed approach are summarized in Figure 1. The flow in homogeneous and heterogeneous saturated porous media and the Nernst-Planck based equations are solved in COMSOL whereas the reactions are solved with PhreeqcRM. A sequential non-iterative operator splitting approach is used for the reactive transport problem. Such approach separates the transport and reactive processes into sequential transport and reaction steps. Contrary to previously proposed coupling schemes, this approach is not based on transport of chemical components but of all chemical species present in the pore water solution. This requirement is crucial for multicomponent ionic transport problems since species-dependent equations and parameters are necessary for the formulation of NernstPlanck equations when using an operator splitting approach. In the proposed Nernst-Planck based coupling the solution of the transport simulator is based on $N+1$ equations in $N+1$ unknowns. The $N+1$ equations are the governing transport equations for the $N$ conservative species and the current balance equation. Under the assumption of electroneutrality, i.e. $\sum_{i} z_{i} C_{i}=0$, and no net electric current the latter equation can be written as:

$$
\nabla \cdot\left(F \sum_{i} z_{i} \boldsymbol{J}_{i}\right)=0
$$

The $N+1$ unknowns are the concentrations of the $N$ transported species and the electrostatic potential that is explicitly computed by solving Eq. 5. The system of equations can be solved by COMSOL as fully coupled, i.e. the equations are solved simultaneously or with a segregated approach. In this study we selected the segregated approach which is less memory intensive but requires smaller time steps to ensure convergence. 
The main features of the proposed coupling approach that are implemented in the coupling scripts include: (i) definition of different aqueous solutions in different portions of the multidimensional COMSOL domain; (ii) automatic definition and update of boundary conditions; (iii) charge balance of all the charged species in solution to maintain electroneutrality in every point of the simulation domain; (iv) amendment of the concentrations after the transport step to avoid negative values that may occur in presence of sharp fronts; (v) flexibility to transfer different variables from PhreeqcRM calculation to COMSOL by use of the PHREEQC's "selected_output" keyword. Besides the transfer of species' concentrations, the latter feature is used to transfer the calculated activity coefficients from PhreeqcRM to COMSOL. This is done by means of a COMSOL dummy physics allowing to store the values of $\gamma_{i}$ in the domain and to calculate the contributions to the multicomponent ionic diffusion and dispersive fluxes from the spatial gradients of the activity coefficients (Eq. 2 and Eq. 3). The length of the coupling step is set by the user depending on the problem under examination, whereas the internal time stepping for transport and reactions are automatically selected by COMSOL and PhreeqcRM to fulfill convergence. The selection of a small time step for the coupling between the two codes is recommended to minimize the operator-splitting error. In the presence of sharp concentration fronts small negative values might occur in some nodes of the domain at the end of the transport step. As suggested in previous studies adopting an operator splitting approach to couple transport and geochemical codes (e.g., Wissmeier and Barry, 2011; Nardi et al., 2014; Parkhurst and Wissmeier, 2015), an amendment is required to correct for possible negative concentrations at the end of the transport step. The amendment (point (iv) above) implemented in NP-Phreeqc is carried out by setting the negative concentrations to the values in such nodes at the previous time step.

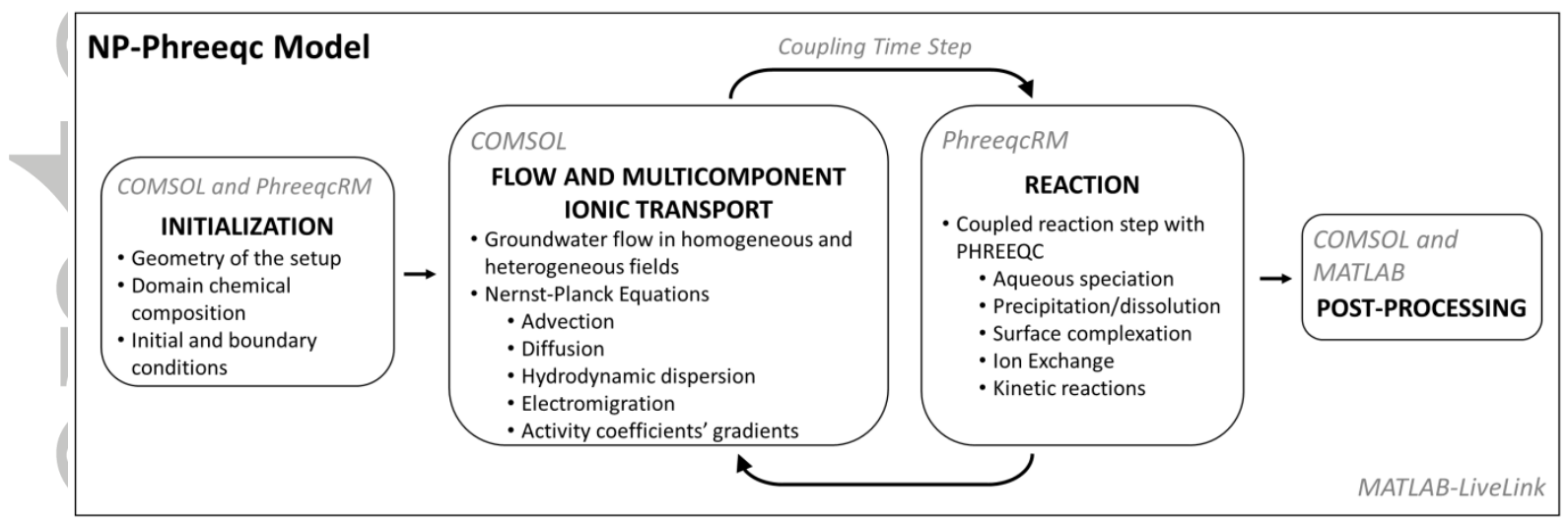

Figure 1. Schematic flow chart of the proposed COMSOL-PhreeqcRM coupling for NernstPlanck based transport of charged species.

2.2 Multidimensional setups: benchmarks and flow-through experiments

Simulations of multicomponent ionic transport with the proposed coupling scheme, based on the Nernst-Planck description of diffusive/dispersive fluxes, are tested in different setups. The outcomes are benchmarked against analytical solutions, numerical simulations, and highresolution experimental datasets of multicomponent ionic transport under flow-through conditions. A schematic representation of the considered 1-D, 2-D and 3-D domains is provided in Figure 2. 
(a) 1-D column

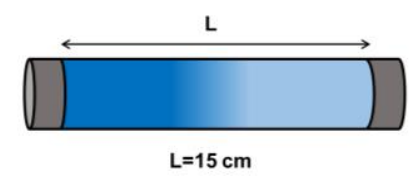

(c) 2-D heterogeneous medium

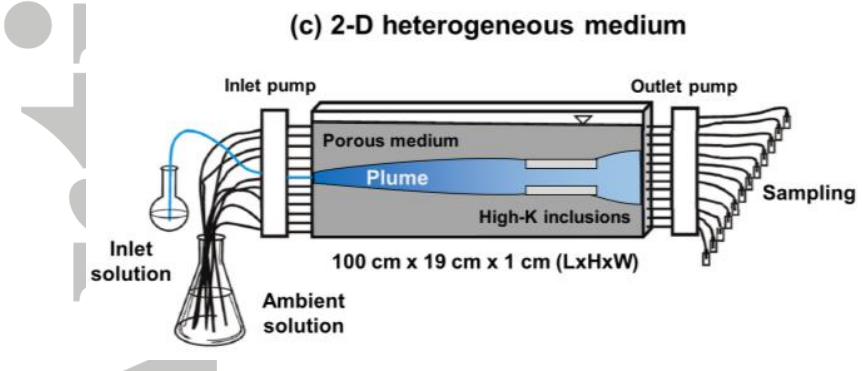

(b) 2-D homogeneous medium

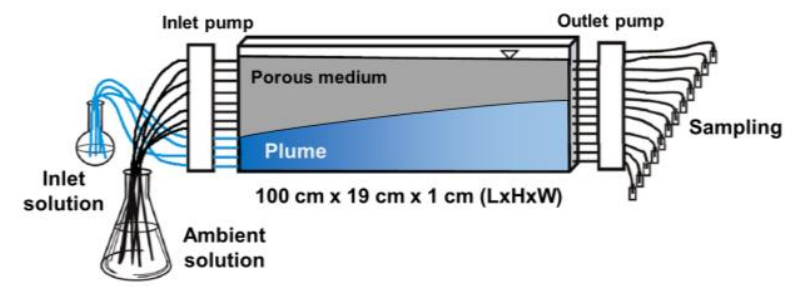

(d) 3-D homogeneous medium

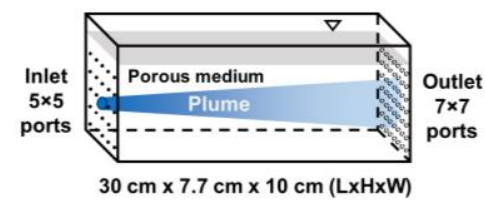

Figure 2. Setups considered for multicomponent ionic transport: (a) 1-D column; (b) 2-D homogeneous medium; (c) 2-D heterogeneous medium; (d) 3-D homogeneous medium.

1-D simulations of multicomponent diffusion of ionic admixtures in pure water have been performed and compared with analogous simulations with PHREEQC. This initial test allowed us to directly assess the capability of the coupling and, in particular, the impact of the ionic strength, activity coefficients and their spatial gradients on the multicomponent ionic fluxes.

In 2-D setups the capabilities of the proposed modeling approach are compared with experimental data from the study of Coulombic interactions during advection-dominated transport of charged species in saturated porous media. First, we consider transport of acidic plumes in a quasi two-dimensional flow-through setup filled with a homogeneous porous medium. Acidic plumes are continuously injected at the bottom of the inlet boundary and deionized water was injected from the remaining inlet ports (Figure 2b). $\mathrm{HCl}$ plumes ( $\mathrm{pH} 4$ ) in different solutions with $\mathrm{MgCl}_{2}$ were used to test the effect of electrochemical cross coupling on the migration of diffusive/dispersive $\mathrm{pH}$ fronts. Details on the flow-through experiments are given by Muniruzzaman and Rolle (2015). A similar scenario with the same geometry and boundary conditions is considered for transport of $\mathrm{H}_{2} \mathrm{SO}_{4}$ in solution with $\mathrm{MgSO}_{4}$. This problem was selected to assess the importance of aqueous speciation reactions, the formation of aqueous complexes and the transport of all primary and secondary species in multicomponent ionic transport. No experimental dataset is available for this scenario and the 2-D steady-state results are compared with the outcomes of 1-D transient multicomponent ionic dispersion simulations with PHREEQC.

Two-dimensional simulations are also performed in heterogeneous porous media. The considered experimental setup is depicted in Figure $2 \mathrm{c}$ and a detailed description was presented in a previous study (Muniruzzaman et al., 2014). Two high-permeability inclusions with coarse grain size are embedded in the porous matrix and cause flow focusing and enhanced mixing between the plume and the surrounding solution (Werth et al., 2006; Rolle et al., 2009). In a first experiment, a $\mathrm{NaCl}$ plume was continuously injected from the center of the inlet boundary whereas deionized water was injected from the surrounding inlet ports. A second experiment was performed by injecting a $\mathrm{MgCl}_{2}$ plume in a $\mathrm{NaBr}$ background electrolyte solution. The highresolution measurements ( $5 \mathrm{~mm}$ spacing) of cations and anions at the outlet are used to test the 
performance of the proposed NP-Phreeqc model for multicomponent transport in heterogeneous porous media.

We also investigate multicomponent ionic transport in 3-D setups. First, we compare the Nernst-Planck based approach for a strong 1:1 electrolyte with an analytical solution for an uncharged species. To this end, we consider continuous injection of a $\mathrm{NaCl}$ plume in pure water in a three-dimensional flow-through domain. Furthermore, new experiments have been performed in this study to explore and document multicomponent ionic transport in fully threedimensional porous media. The 3-D experimental setup was described in previous studies of tracer transport in homogeneous, heterogeneous isotropic and anisotropic porous media (Ye et al., 2015a-c). Therefore, here we only briefly summarize the main features of the setup and of the experiments that we performed. The flow-through experiments are carried out in a threedimensional flow-through chamber with inner dimensions of $30 \mathrm{~cm} \times 10 \mathrm{~cm} \times 7.7 \mathrm{~cm}$ (Figure $2 \mathrm{~d}$ ). The chamber is made of acrylic glass and has 25 equally spaced ( $1.54 \mathrm{~cm}$ spacing) injection ports at the inlet and 49 equally spaced $(1.1 \mathrm{~cm}$ spacing) extraction ports at the outlet. Syringe needles piercing rubber septa are used to inject and extract the pore water solutions at the inlet and outlet ports. High-precision peristaltic pumps with 24 channels (Ismatec, Glattbrugg, Switzerland) are used to establish steady-state flow conditions. Homogeneous quartz sand (Euroquarz, Germany) with grain diameter of 1.0-1.5 mm was used as porous medium. The sand was filled following a wet-packing procedure using ultrapure Milli-Q water (Labostar 1-DI, Evoqua, USA) to avoid air entrapment in the porous medium (Haberer et al., 2012). The porosity of the packed medium was 0.4 . The experiments were performed at an average flow velocity of $0.5 \mathrm{~m} /$ day. After establishing steady-state flow conditions, a solution of magnesium chloride $(0.30 \mathrm{mM})$ was injected through the central inlet port and, in two different experiments, either Milli-Q water or a solution of $\mathrm{NaBr}(1.70 \mathrm{mM})$ were injected from the surrounding inlet ports. To ensure the achievement of steady-state transport conditions, we flushed three pore volumes before starting sampling. Samples were collected at the 49 extraction ports at the outlet $(7 \times 7$ array) and the ion concentrations were measured with ion-chromatography for the anions (Dionex ICS-1500, Thermo Scientific, USA) and with ICP-MS (Agilent 7700 Series, Agilent Technologies, Japan) for the cations.

Finally, we tested the capability of the coupled model to simulate 3-D reactive transport. To this end, we considered a column setup in which advection, multicomponent ionic dispersion and ion exchange take place. The chemistry of the problem is analogous to the one described in the example 11 of the PHREEQC-3 manual (Parkhurst \& Appelo, 2013) and entails an exchanger column initially in equilibrium with a solution containing sodium and potassium nitrate, flushed by a calcium chloride solution. The transport and ion exchange problem is resolved considering its full three dimensional representation, by solving Eq. 4 with the proposed NP-Phreeqc model. The outcomes are compared with the solution of the same problem in 1-D using PHREEQC.

Table 1 provides an overview of the problems selected to illustrate the proposed modeling approach. The table includes the description of the key features of each problem and the relative benchmarks that are based on modeling and/or experimental datasets. 
Table 1. Overview of Nernst-Planck based transport simulations with the proposed COMSOLPhreeqcRM coupling and relative benchmarks.

\begin{tabular}{|c|c|c|}
\hline Problem & Description and key features & Benchmark \\
\hline $\begin{array}{l}\text { 1-D multicomponent ionic } \\
\text { diffusion }\end{array}$ & $\begin{array}{l}\text { - Diffusion of an electrolyte mixture in pure } \\
\text { water at different concentrations } \\
\text { - Evaluation of the impact of the flux } \\
\text { contribution due to the gradient of activity } \\
\text { coefficients }\end{array}$ & $\begin{array}{l}\text { 1-D diffusion } \\
\text { simulations with } \\
\text { PHREEQC }\end{array}$ \\
\hline $\begin{array}{l}\text { 2-D multicomponent ionic } \\
\text { dispersion in } \\
\text { homogeneous setup }\end{array}$ & $\begin{array}{l}\text { - Flow-through transport of a } \mathrm{HCl} \text { plume in } \\
\text { different ionic admixtures: impact of charge } \\
\text { interactions on pH front propagation } \\
\text { - Flow-through transport of a } \mathrm{H}_{2} \mathrm{SO}_{4} \text { plume in a } \\
\mathrm{MgSO}_{4} \text { ionic admixture: impact of speciation } \\
\text { and aqueous complexes }\end{array}$ & $\begin{array}{l}\text { 2-D Experimental } \\
\text { dataset }^{\mathrm{a}} \\
\text { 1-D transient dispersion } \\
\text { simulations with } \\
\text { PHREEQC }\end{array}$ \\
\hline $\begin{array}{l}\text { 2-D multicomponent ionic } \\
\text { dispersion in } \\
\text { heterogeneous setup }\end{array}$ & $\begin{array}{l}\text { - Flow-through transport of a } \mathrm{NaCl} \text { plume in } \\
\text { Milli-Q water and of a } \mathrm{MgCl}_{2} \text { plume in a } \mathrm{NaBr} \\
\text { background solution in a spatially variable flow } \\
\text { field }\end{array}$ & $\begin{array}{l}\text { 2-D Experimental } \\
\text { dataset }^{\mathrm{b}}\end{array}$ \\
\hline $\begin{array}{l}\text { 3-D multicomponent ionic } \\
\text { transport }\end{array}$ & $\begin{array}{l}\text { - Comparison with an analytical solution to } \\
\text { verify the computation of Coulombic } \\
\text { interactions in three dimensions for transport of } \\
\text { a } \mathrm{NaCl} \text { plume in pure water } \\
\text { - Flow-through transport of a } \mathrm{MgCl}_{2} \text { plume in } \\
\text { Milli-Q water and in a } \mathrm{NaBr} \text { background } \\
\text { solution }\end{array}$ & $\begin{array}{l}\text { 3-D flow-through } \\
\text { experiments }\end{array}$ \\
\hline $\begin{array}{l}\text { 3-D multicomponent ionic } \\
\text { transport and ion } \\
\text { exchange }\end{array}$ & $\begin{array}{l}\text { Example of reactive transport in a three- } \\
\text { dimensional domain }\end{array}$ & $\begin{array}{l}\text { 1-D simulations with } \\
\text { PHREEQC }\end{array}$ \\
\hline
\end{tabular}

\section{Results and Discussion}

3.1 1-D multicomponent ionic diffusion

A one-dimensional problem of multicomponent ionic diffusion is considered to test the proposed NP-Phreeqc model and to compare its outcomes with PHREEQC simulations. A $12 \mathrm{~cm}$ long column (Figure 2a) is initially occupied for the left half by a solution containing $\mathrm{HCl}$ and $\mathrm{MgCl}_{2}$ (source zone) whereas the remaining portion of the domain contains pure water. During the simulation the ions diffuse from the source zone to the remaining part of the domain due to their electrochemical potential gradients. Two different scenarios with dilute and more concentrated solutions in the initial source are considered (Table 2). The table also reports the values of aqueous diffusion coefficients. For this example and throughout this work we consider the $D_{a q}$ values for major cations and anions reported by Lasaga (1998) at $\mathrm{T}=18{ }^{\circ} \mathrm{C}$ and corrected for temperature and viscosity at $\mathrm{T}=20^{\circ} \mathrm{C}$. The latter is the temperature at which the flow-through experiments illustrated in the next sections have been performed. The diffusivities of aqueous complexes have been calculated from the limiting molar conductivities with the Nernst-Einstein relationship and using Kohlrausch's law to estimate the conductivity values (Lide, 2005). The 
standard values obtained were also corrected for temperature and viscosity to determine the aqueous diffusivities of complexes at $\mathrm{T}=20{ }^{\circ} \mathrm{C}$.

Table 2. 1-D multicomponent ionic diffusion

Setup geometry and parameters

Length of the 1-D setup [cm]

Number of elements in NP-Phreeqc

12

Source solution (Scenario 1 / Scenario 2):

$\mathrm{HCl}[\mathrm{mM}]$

12000

$\mathrm{MgCl}_{2}[\mathrm{mM}]$

$0.1 / 0.1$

Diffusion coefficients at $\mathrm{T}=20^{\circ} \mathrm{C}$

$1 / 100$

$\mathrm{H}^{+}$

$\mathrm{Mg}^{2+}$

$8.65 \times 10^{-9}$

$\mathrm{Cl}^{-}$

$0.63 \times 10^{-9}$

$1.81 \times 10^{-9}$

During the simulation, the species in the ionic admixtures diffuse from left to right. Their diffusive displacement depends not only on the aqueous diffusivities of the ions at liberated states but also on the Coulombic interactions between the different charged species in solution. The results after 12 hours of simulation are illustrated in Figure 3.
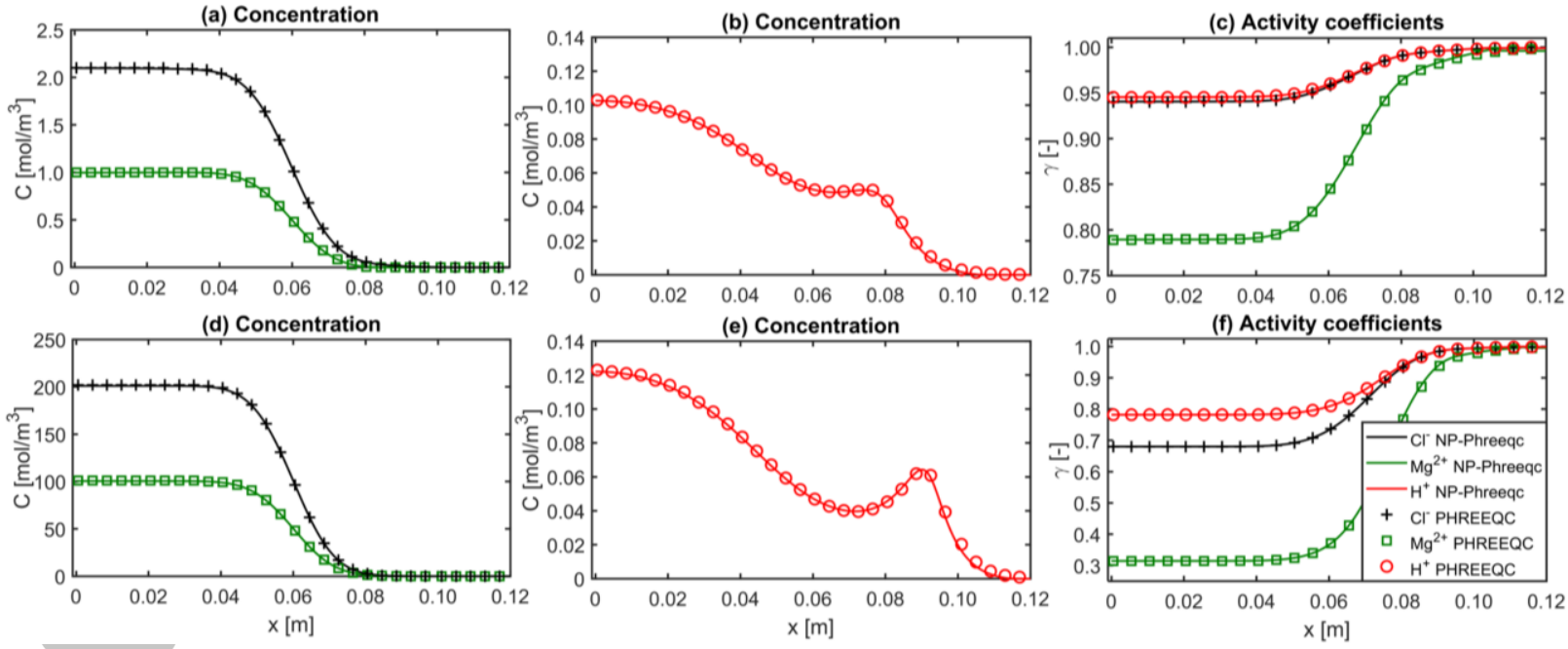

Figure 3. Spatial profiles of ions' concentrations and activity coefficients for 1-D diffusion of a $\mathrm{MgCl}_{2}$ and $\mathrm{HCl}$ solution in pure water at low $(\mathrm{I}=0.0031 \mathrm{M})(\mathrm{a}-\mathrm{c})$ and high $(\mathrm{I}=0.303 \mathrm{M})(\mathrm{d}-\mathrm{f})$ ionic strength.

The major cations and anions $\left(\mathrm{Mg}^{2+}\right.$ and $\left.\mathrm{Cl}^{-}\right)$, present in excess compared to $\mathrm{H}^{+}$in the initial source solution, undergo a similar displacement as shown by the shape of their diffusive profiles (Figure $3 \mathrm{a}$ and $3 \mathrm{~d}$ ). The $\mathrm{H}^{+}$ions (Figure $3 \mathrm{~b}$ and $3 \mathrm{e}$ ) show a more spread concentration profile that is clearly influenced by the interaction with the other ions in the domain, particularly in the right portion where such interactions lead to the formation of a peak in the protons' concentration profiles. Figure 3 (c and f) also shows the values of the activity coefficients of the different ions. Their computation is performed with the expressions implemented in the PHREEQC database (phreeqc.dat): the Trusedell and Jones equation for $\mathrm{Mg}^{2+}$ and $\mathrm{Cl}^{-}$and the 
Debye-Hückel equation for $\mathrm{H}^{+}$, respectively (Appelo and Postma, 2005). The results show an increasing trend of $\gamma_{i}$ in the direction of the diffusive flux, from the source to the pure water solution. Larger values and smaller gradients of $\gamma_{i}$ are obtained in the dilute scenario with lower ionic strength (Figure 3c).

The inspection of the fluxes allows understanding and visualizing the influence of the ions' interactions in the solution. As described in Eq. 2, the three main components that affect the total diffusive displacement of each ion are: the purely diffusive flux, the activity gradient flux and the electromigration flux:
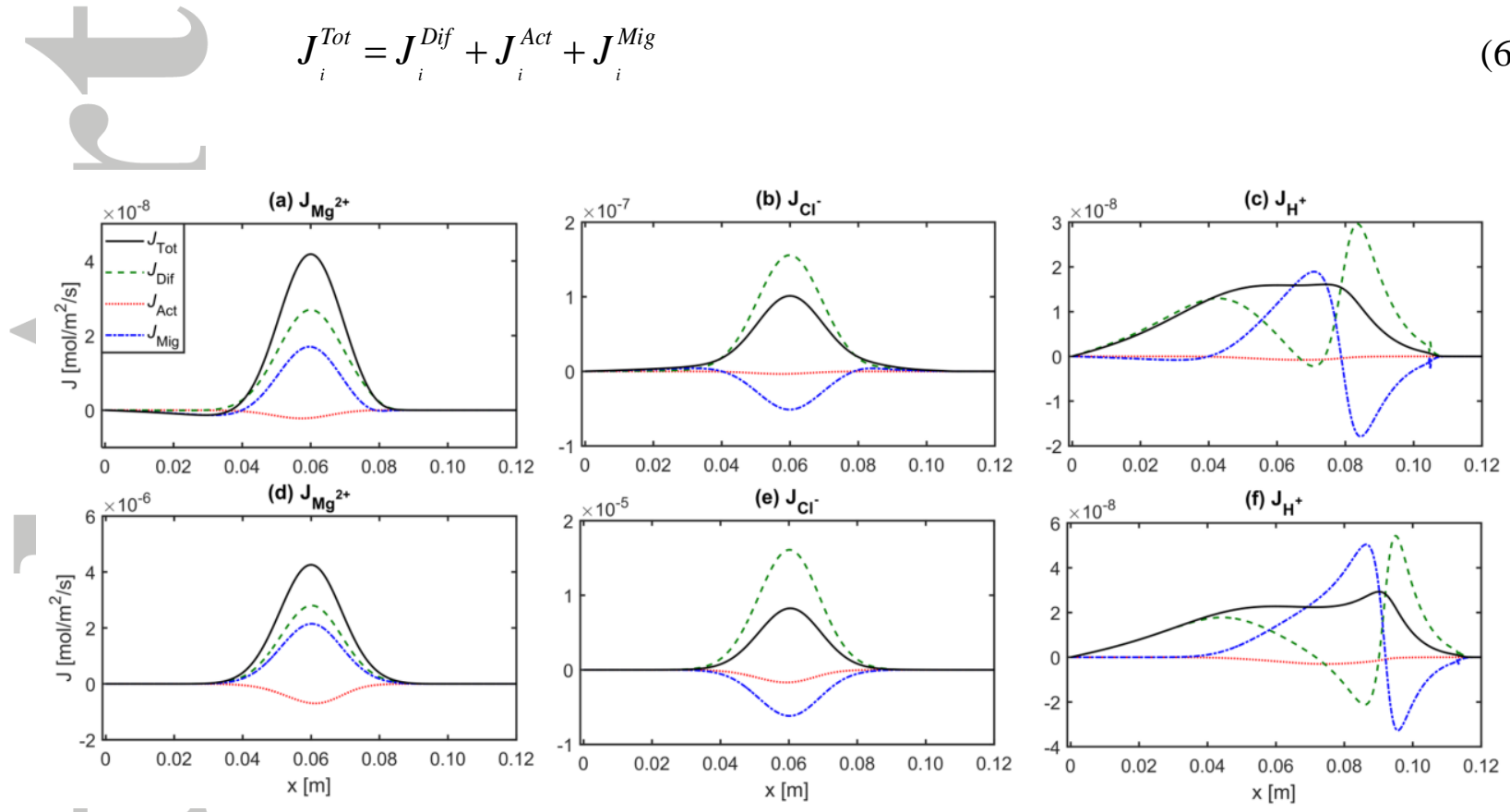

Figure 4. Flux contributions for the 1-D multicomponent diffusion problems: spatial profiles after 12 hours of simulation for the case at low ionic strength (a-c) and at high ionic strength (de).

The results for the two scenarios are shown in Figure 4. The different flux components have distinct contributions to the displacement of the different ions. For instance the electromigration contribution is positive for $\mathrm{Mg}^{2+}$ but negative for $\mathrm{Cl}^{-}$. The displacement of the former is thus enhanced through the Coulombic interactions, whereas the chloride displacement is reduced by the coupling with magnesium. The contribution of the activity gradient flux is negative for all ions: its effect is small in the scenario with dilute solution (Figure 4 a-c), but becomes more relevant in the case at high ionic strength (Figure $4 \mathrm{~d}-\mathrm{f}$ ). Particularly interesting are the flux contributions for the hydrogen ions. For most of the column length both the diffusive and the electromigration fluxes show a positive contribution that enhances the displacement of $\mathrm{H}^{+}$. However, towards the right boundary of the column setup, $J_{H^{+}}^{\mathrm{Mig}}$ decreases and becomes negative (Figures $4 \mathrm{c}$ and $4 \mathrm{f}$ ). This happens because in this outer fringe zone of the domain, the other two ions $\left(\mathrm{Mg}^{2+}\right.$ and $\left.\mathrm{Cl}^{-}\right)$are present only in trace amounts, similar to the concentration of 
$\mathrm{H}^{+}$. This results in a coupling between the displacement of protons and chloride causing a reduction of the total diffusive flux of $\mathrm{H}^{+}$.

\subsection{Multicomponent ionic transport in 2-D homogeneous porous media}

\subsubsection{Transport of acidic plumes: propagation of $\mathrm{pH}$ fronts}

As illustrative examples of multidimensional transport and Coulombic interactions in flow-through systems we consider the experimental scenarios studied by Muniruzzaman and Rolle (2015). The setup and boundary conditions are schematically illustrated in Figure $2 \mathrm{c}$ and an overview of the parameters from the experiments and used in the multicomponent transport simulations are summarized in Table 3 . The chemistry of the problem is analogous to the 1-D setup illustrated above, consisting of an $\mathrm{HCl}$ plume in solutions with different concentrations of $\mathrm{MgCl}_{2}\left(\mathrm{C}_{\mathrm{H}+} / \mathrm{C}_{\mathrm{Mg} 2+} \sim 1\right.$ and $\mathrm{C}_{\mathrm{H}+} / \mathrm{C}_{\mathrm{Mg} 2+} \sim 0.01$, respectively) continuously injected at the bottom of the flow-through chamber.

Table 3. Multicomponent ionic transport of acidic plumes in 2-D flow-through setups.

\section{Experimental settings}

Flow-through domain $(L \times H)[\mathrm{cm}]$

$100 \times 12$

Source thickness [cm]

Number of elements in 2-D NP-Phreeqc

19782

Grain diameter of the porous matrix, $d$ [mm]

Hydraulic conductivity, $K[\mathrm{~m} / \mathrm{s}]$

$1.00-1.50$

Average horizontal flow velocity, $v$ [m/day]

$1.27 \times 10^{-2}$

Ayerage porosity, $n[-]$

0.8

0.41

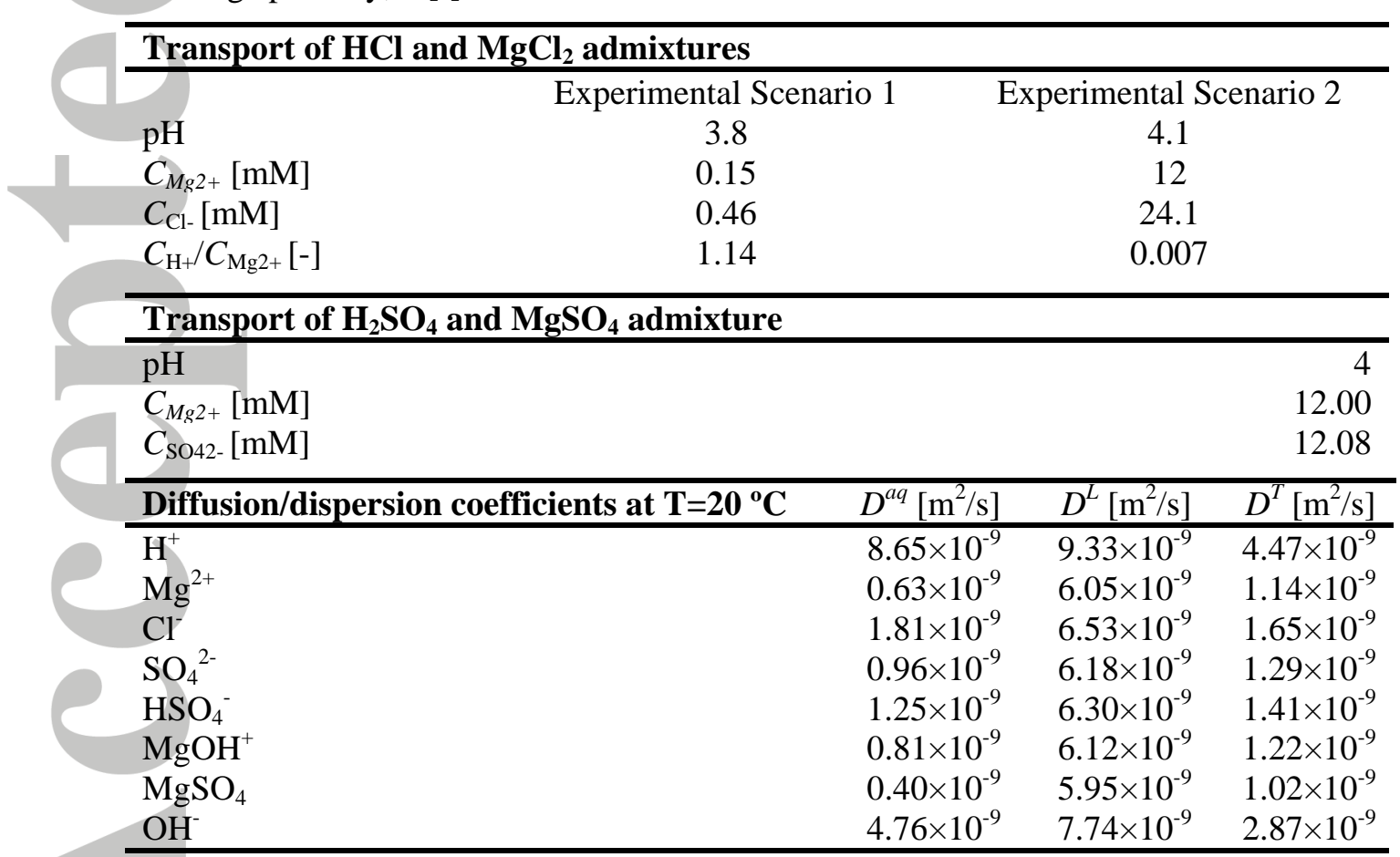

The experiments were performed under advection-dominated conditions, thus a NernstPlanck description of the dispersive fluxes (Eq. 3) is necessary to describe transport and interactions between the charged species. To this end, an accurate parameterization of local 
hydrodynamic dispersion is required. In this study we use the empirical correlations obtained from a number of flow-through experiments performed to accurately characterize longitudinal (Guedes de Carvalho \& Delgado, 2005; Delgado, 2006) and transverse (Chiogna et al., 2010; Rolle et al., 2012) dispersion coefficients in granular porous media:

$$
\begin{aligned}
& D_{i}^{L}=D_{i}^{P}+\frac{1}{2} v d \\
& D_{i}^{T}=D_{i}^{P}+D_{i}^{a q}\left(\frac{P e_{i}^{2}}{P e_{i}+2+4 \delta^{2}}\right)^{\beta}
\end{aligned}
$$

where $D_{i}^{a q}$ is the aqueous diffusion coefficient, $D_{i}^{P}$ is the pore diffusion coefficient taking into account that the aqueous diffusivity is reduced due to the tortuosity of the porous medium and approximated as $D_{i}^{P} \approx n D_{i}^{a q}, d$ is the average grain size diameter of the granular packing used in the experiments and $P e_{i}=v d / D_{i}^{a q}$ is the grain Péclet number of species $i$ and $v$ is the seepage velocity component along the main flow direction. $\delta$ denotes the ratio between the length of a pore channel to its hydraulic radius and $\beta$ is an empirical exponent that accounts for the effect of incomplete mixing in the pore channels. The values $\delta=6.2$ and $\beta=0.5$ were determined in previous experimental and pore-scale modeling studies (Rolle et al., 2012; Hochstetler et al., 2013). Since transport of the acidic plumes is studied under steady-state conditions, the transverse dispersion coefficient (Eq. 8) and the coupling between transverse dispersive fluxes of the different charged species, control their displacement in the porous medium and, thus, the ions' concentrations measured at the outlet ports. The longitudinal dispersion coefficient is less important for this problem since, under continuous injection and steady-state transport, the concentration gradients along the flow direction are very small and the contribution of the longitudinal dispersion fluxes can be safely neglected (Cirpka et al., 2011 and 2015).

The outcomes of the simulations and the comparison with the experimental results are illustrated in Figure 5. The panels on the left show the computed 2-D distribution of the different ionic species in the flow-through domain. The panels on the right show the spatial profiles at the outlet. The different ionic admixtures remarkably influence the lateral propagation of the $\mathrm{pH}$ fronts. In the scenario with small $\mathrm{MgCl}_{2}$ concentrations $\left(\mathrm{C}_{\mathrm{H}+} / \mathrm{C}_{\mathrm{Mg} 2+} \sim 1\right)$ the different ions show a distinct pattern of transverse displacement with spreading of the common anion $\mathrm{Cl}^{-}$larger than the one of $\mathrm{Mg}^{2+}$ and smaller than $\mathrm{H}^{+}$. In the case with higher $\mathrm{MgCl}_{2}$ concentrations $\left(\mathrm{C}_{\mathrm{H}+} / \mathrm{C}_{\mathrm{Mg} 2+} \sim 0.01\right)$ the major cation and anion $\left(\mathrm{Mg}^{2+}\right.$ and $\left.\mathrm{Cl}^{-}\right)$show an almost overlapping profile, whereas the profile of $\mathrm{H}^{+}$is significantly more spread and, thus, the lateral propagation of the $\mathrm{pH}$ plume is enhanced compared to the previous scenario. The Nernst-Planck description of the dispersive fluxes implemented with the proposed NP-Phreeqc model shows an excellent agreement with the high-resolution experimental data. The modeling outcomes are the results of purely forward simulations performed with the data provided in Table 3 and without any fitting parameter.

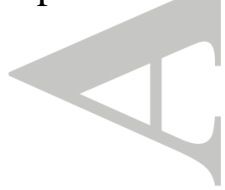



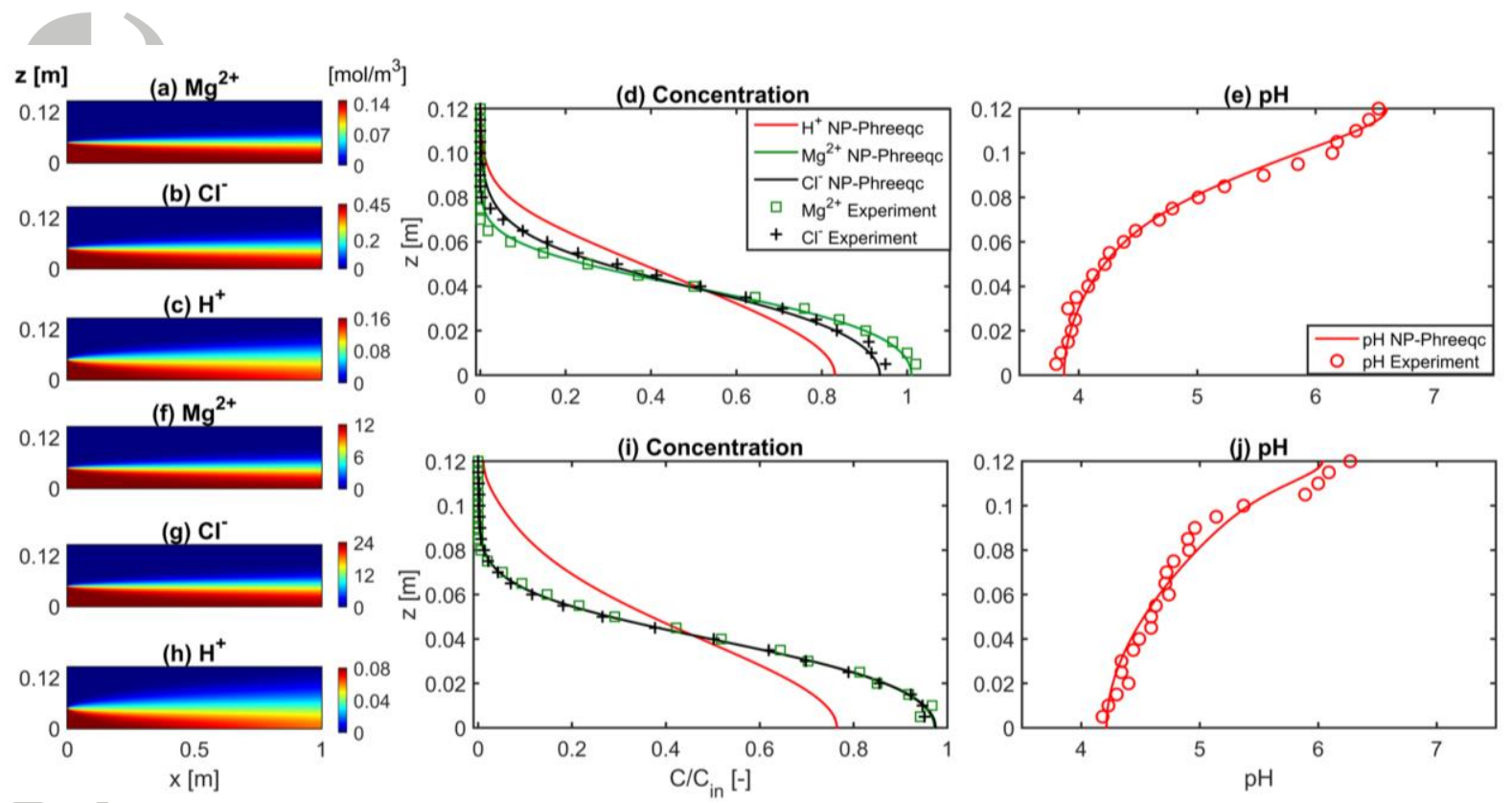

Figure 5. 2-D maps of ion concentrations and measured vs. simulated concentration and $\mathrm{pH}$ profiles at the outlet of the flow-through setups for the cases $\mathrm{C}_{\mathrm{H}+} / \mathrm{C}_{\mathrm{Mg} 2+} \sim 1$ (a-e) and $\mathrm{C}_{\mathrm{H}+} / \mathrm{C}_{\mathrm{Mg} 2+} \sim 0.01(\mathrm{f}-\mathrm{j})$.

\subsubsection{Transport of acidic plumes: the effect of speciation and aqueous complexes}

Another example of transport of acidic plumes considered in this study is the continuous release of an $\mathrm{H}_{2} \mathrm{SO}_{4}$ and $\mathrm{MgSO}_{4}$ admixture. The geometry and boundary conditions are the same as those for transport of $\mathrm{HCl}$ and $\mathrm{MgCl}_{2}$ described in the previous section, but now the $\mathrm{H}_{2} \mathrm{SO}_{4}$ plume was selected to illustrate the effects of aqueous speciation and ion complexes. No experimental data are available for this transport problem to assess the performance of the NPPhreeqc simulations. However, the 2-D multicomponent ionic simulations are benchmarked with an equivalent $1-D$ problem solved with PHREEQC. In fact, in a uniform flow field and neglecting the contribution of the longitudinal dispersive term, the governing steady-state transport equation in 2-D is analogous to a 1-D transient dispersive problem (Maier \& Grathwohl, 2006; Van Breukelen \& Rolle, 2012). The parameters for the 1D and 2D setups are reported in Table 3. Contrary to the case of $\mathrm{HCl}$ transport in $\mathrm{MgCl}_{2}$ solution where aqueous speciation was dominated by the free ions, for transport of an admixture of $\mathrm{H}_{2} \mathrm{SO}_{4}$ and $\mathrm{MgSO}_{4}$, the formation of ion complexes plays an important role. Solute species that are quantitatively relevant for this problem include $\mathrm{HSO}_{4}{ }^{-}, \mathrm{MgSO}_{4}$ and $\mathrm{MgOH}^{+}$. Thus, for this problem it is particularly important to transport all the species as done by PHREEQC for 1-D transport and by the proposed COMSOL-PhreeqcRM coupling for multidimensional problems. The comparison between the two codes, including the different transported species, is reported in Figure 6a. The results show a very good match between the 1-D and 2-D simulations of multicomponent ionic transport for both free ions and aqueous complexes. Figure $6 \mathrm{~b}$ shows two-dimensional representations of the distinct terms of the Nernst-Planck fluxes, which are formally identical to Eq. 5, but consider the contribution of transverse hydrodynamic dispersion in the flow-through setup: 


$$
J_{i}^{T o t}=J_{i}^{\text {Disp }}+J_{i}^{A c t}+J_{i}^{M i g}
$$

The distinct plots (Figure 6b) allow visualizing the different flux contributions for sulfate transport. It is interesting to notice that $J^{A c t}$ has a relevant negative impact to the overall dispersive flux. In fact, due to the importance of aqueous complexation, the activity coefficients' gradients are particularly steep at the fringe of the plume, where the plume's electrolyte solution mixes with the surrounding pure water.
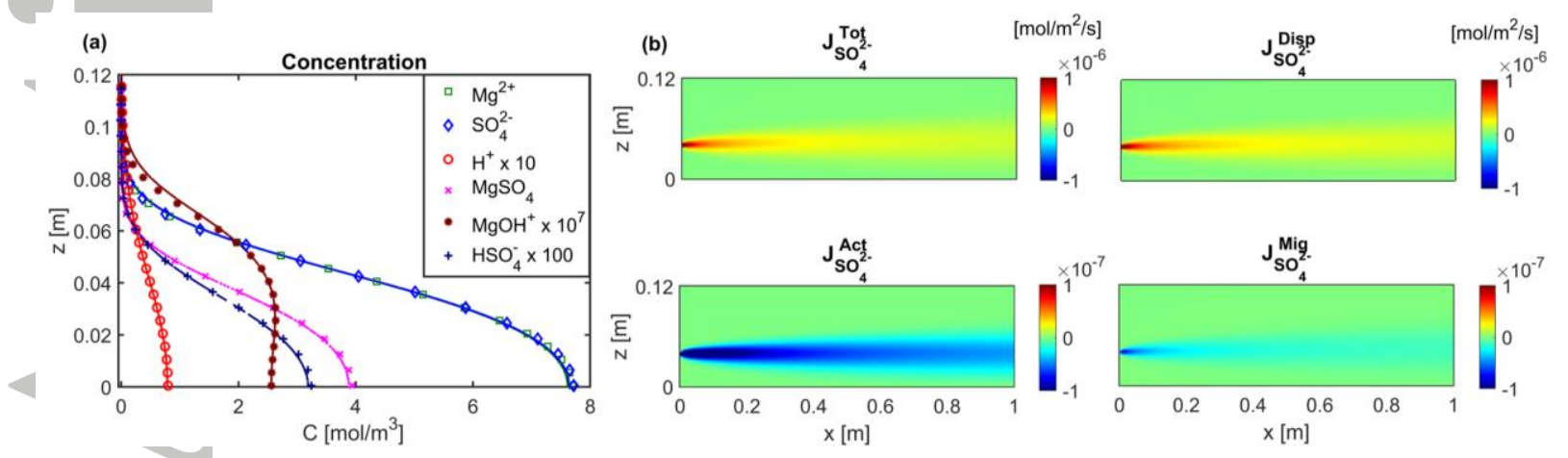

Figure 6. Multicomponent ionic transport of a $\mathrm{H}_{2} \mathrm{SO}_{4}$ and $\mathrm{MgSO}_{4}$ admixture in a 2-D flowthrough setup. Comparison of 2-D NP-Phreeqc steady-state simulations (lines) with 1-D transient PHREEQC simulations (symbols) (a). Spatial maps of the transverse dispersive fluxes of sulfate (b); in the computation of the flux contributions the direction from the plume core to the plume fringe (i.e., from high to low concentration values) is considered positive.

3.3 Multicomponent ionic transport in 2-D heterogeneous porous media

To test the capability of the proposed approach to simulate multicomponent ionic transport in non-uniform flows we considered a flow-through experiment performed in a previous study (Muniruzzaman et al., 2014). Figure 2c illustrates the experimental setup which has the same dimensions and geometry of the system described in the previous section. A heterogeneous porous medium was obtained by embedding two high-permeability inclusions made with coarse grain sizes $\left(d=2.00-2.50 \mathrm{~mm}, K=4.12 \times 10^{-2} \mathrm{~m} / \mathrm{s}\right)$ in a matrix of finer material $\left(d=1.00-1.50 \mathrm{~mm}, K=1.27 \times 10^{-2} \mathrm{~m} / \mathrm{s}\right)$. The inclusions were $20 \mathrm{~cm}$ long and $1 \mathrm{~cm}$ thick and were placed $70 \mathrm{~cm}$ from the inlet and $1.5 \mathrm{~cm}$ from the plume's centerline. Dilute solutions of salt plumes were injected from the two central inlet ports whereas Milli-Q water or background electrolyte solutions were continuously injected from the surrounding 22 inlet ports. A first experiment was performed by injecting a $\mathrm{NaCl}$ plume $(0.8 \mathrm{mM})$ in Milli-Q water. The plume was transported in the heterogeneous flow field, and measurements were taken at the outlet ports. The outcomes of this experiment are reported in Figure 7a. The vertical profiles show a clearly non-Gaussian shape with peak concentrations showing a lower transverse spreading, whereas the lower concentrations are remarkably more spread due to the enhanced mixing through flowfocusing in the high-permeability inclusions. Despite the diffusivity of chloride $\left(D_{C l}^{a q}=1.81 \times 10^{-9}\right.$ $\mathrm{m}^{2} / \mathrm{s}$ at $\left.20^{\circ} \mathrm{C}\right)$ is significantly higher than the one of sodium $\left(D_{\mathrm{Na}}^{a q}=1.20 \times 10^{-9} \mathrm{~m}^{2} / \mathrm{s}\right.$ at $\left.20^{\circ} \mathrm{C}\right)$, the displacement of the ions in the flow-through system is coupled and the experimental data show an analogous pattern for $\mathrm{Na}^{+}$and $\mathrm{Cl}^{-}$. The outcomes of the multicomponent ionic simulations in 
the heterogeneous setup performed with NP-Phreeqc allow reproducing the observed behavior and yield identical profiles of sodium and chloride. A second experiment was performed injecting a $\mathrm{MgCl}_{2}$ plume $(0.44 \mathrm{mM})$ in a background solution of $\mathrm{NaBr}(1.70 \mathrm{mM})$. In these conditions a clear separation between the profiles of $\mathrm{Mg}^{2+}$ and $\mathrm{Cl}^{-}$was observed. Such behavior depends on the higher concentration of the background electrolyte ions that ensure the fulfillment of the electroneutrality constraint and allow a distinct displacement of the plume ions. In fact, $\mathrm{Mg}^{2+}$ and $\mathrm{Cl}^{-}$displace almost at their liberated state, with their specific diffusive/dispersive properties. Also in this case the outcomes of the purely forward NP-Phreeqc simulations yields satisfactory results and show a clear separation of the plume cations and anions as observed in the flow-through experiments.
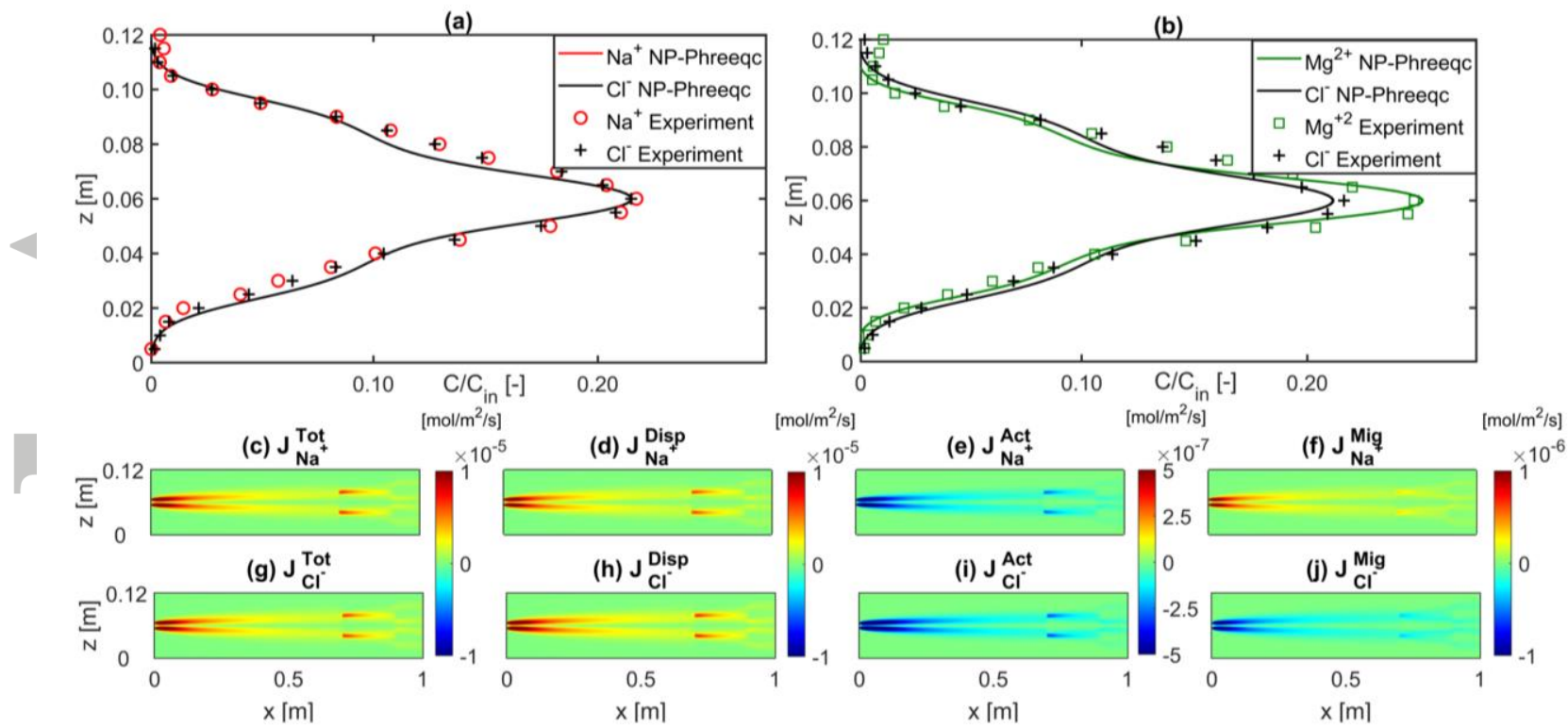

Figure 7. Transverse concentration profiles at the outlet of the 2-D heterogeneous setup for transport of $\mathrm{NaCl}$ in Milli-Q water (a) and $\mathrm{MgCl}_{2}$ in a $\mathrm{NaBr}$ background solution (b). Maps of the different contributions of the multicomponent ionic transverse dispersive fluxes for transport of $\mathrm{NaCl}$ in Milli-Q water (c-j). The direction from the plume core to the plume fringe is considered positive for the computed fluxes.

Figure 7 also shows the maps of the transverse dispersive fluxes in the 2-D heterogeneous domain for the case of continuous injection of the 1:1 electrolyte plume $(\mathrm{NaCl})$. The total dispersive fluxes of sodium and chloride are the same and show high values close to the inlet and in the high-permeability inclusions where the flow is focused and lateral mixing is enhanced. However, the components of the total dispersive flux are different. In fact, whereas the dispersive flux of sodium is enhanced by the coupling with chloride as shown by its positive electromigration contribution, the displacement of $\mathrm{Cl}^{-}$is limited by the electrostatic coupling with $\mathrm{Na}^{+}$(i.e., negative electromigration contribution for chloride). Notice that, differently from the example illustrated in Figure 6, for this scenario the dispersive flux term due to the gradient of the activity coefficients has a smaller contribution compared to the other flux components. 
3.4 Conservative and reactive multicomponent ionic transport in 3-D setups

\subsubsection{3-D Analytical solution and numerical simulation of $\mathrm{NaCl}$ transport}

Analytical solutions for groundwater solute transport problems are available for different geometries and boundary conditions (e.g., Van Genuchten et al., 1991; Leij et al., 2013) and are important for benchmarking numerical codes. Here we compare the Nernst-Planck based formulation for charged species transport with an analytical solution for a fully three dimensional problem. We consider a 3-D domain representative of the flow-through setup illustrated in Figure $2 \mathrm{~d}$. A conservative tracer with concentration $C_{0}$ is continuously released from a rectangular source of width $Y$ and thickness $Z$, whereas pure water is injected from the remaining portion of the inlet boundary:

$$
C(x=0, y, z)= \begin{cases}C_{0} & \forall|y|<\frac{Y}{2} \cap \forall|z|<\frac{Z}{2} \\ 0 & \forall|y| \geq \frac{Y}{2} \cup \forall|z| \geq \frac{Z}{2}\end{cases}
$$

The analytical solution of the three-dimensional, steady-state transport problem reads as (Domenico and Palciauskas, 1982):

$$
\begin{aligned}
& C(x, y, z)=\frac{1}{4} C_{0}\left\{\operatorname{erf}\left[\frac{y+Y / 2}{2\left(D^{T} x / v\right)^{1 / 2}}\right]-\operatorname{erf}\left[\frac{y-Y / 2}{2\left(D^{T} x / v\right)^{1 / 2}}\right]\right\} \\
& \left\{\operatorname{erf}\left[\frac{z+Z / 2}{2\left(D^{T} x / v\right)^{1 / 2}}\right]-\operatorname{erf}\left[\frac{z-Z / 2}{2\left(D^{T} x / v\right)^{1 / 2}}\right]\right\}
\end{aligned}
$$

The numerical simulation of multicomponent ionic transport is performed for the continuous release of a single 1:1 electrolyte $(\mathrm{NaCl})$ in pure ambient water in the same 3-D setup. Under these conditions, the electrostatic interactions couple the displacement of the cation $\left(\mathrm{Na}^{+}\right)$and the anion $\left(\mathrm{Cl}^{-}\right)$and lead to an identical movement of the two species. Thus, although the salt $(\mathrm{NaCl})$ fully ionizes in the aqueous solution and the two ions $\left(\mathrm{Na}^{+}\right.$and $\left.\mathrm{Cl}^{-}\right)$have different mobilities, they travel as a single species due to the electromigration coupling to maintain electroneutrality. Hence, for this particular case, the diffusion (and hydrodynamic dispersion) of these two ions can be characterized by a single coefficient of the virtual salt species (e.g., Cussler, 2009):

$$
D_{\mathrm{NaCl}}=\frac{\left|z_{\mathrm{Na}^{+}}\right|+\left|z_{\mathrm{Cl}^{-}}\right|}{\left|z_{\mathrm{Na}^{+}}\right| / D_{\mathrm{Cl}^{-}}^{a q}+\left|z_{\mathrm{Cl}^{-}}\right| / D_{\mathrm{Na}^{+}}^{a q}}
$$

where $D_{\mathrm{NaCl}}$ is the combined diffusion coefficient of the electrolyte. $z_{\mathrm{Na}^{+}}, z_{\mathrm{Cl}^{-}}$and $D_{\mathrm{Na}^{+}}^{a q}$, $D_{C^{-}}^{a q}$ are the charge and the aqueous diffusion coefficients of $\mathrm{Na}^{+}$and $\mathrm{Cl}^{-}$, respectively. The value for $D_{\mathrm{NaCl}}$ at $20^{\circ} \mathrm{C}$ is obtained from those of the free ions (Eq. 11) and is equal to $1.44 \times 10^{-9} \mathrm{~m}^{2} / \mathrm{s}$. For this case of transport of a strong electrolyte in pure water, the electrostatic ionic interactions reduce the multicomponent ionic transport problem to a single-species conservative transport problem. Thus, in this case, the outcomes of the 3-D multicomponent ionic transport numerical 
model can be directly compared with the results of the 3-D analytical solution (Eq. 9). Both numerical and analytical models simulate transport in a flow-through system with a seepage velocity of $0.5 \mathrm{~m} /$ day and considering continuous release from a square source $(Y=Z=1 \mathrm{~cm})$. In the numerical model the 3 -D domain $(30 \mathrm{~cm} \times 8 \mathrm{~cm} \times 8 \mathrm{~cm})$ was discretized into 209265 elements. The hydrodynamic dispersion coefficients used as input for the analytical and numerical simulations are computed according to Eqs. 6-7. The value of $D^{T}$ for the virtual salt species as conservative tracer in the analytical solution are calculated using the salt diffusion coefficient (Eq. 11). In the NP-Phreeqc model, instead, the self-diffusion coefficients of the individual ions are used as input values and the hydrodynamic dispersion coefficients are computed (Eqs. 6-7) for the cation and anion at liberated state. The dispersive fluxes of $\mathrm{Na}^{+}$and $\mathrm{Cl}^{-}$are coupled in the multicomponent ionic formulation of the numerical model (Eq. 3 and Eq. 4). The results of the 3-D simulations and the comparison between the analytical solution and the multicomponent ionic transport model are reported in Figure 8. The steady-state plume and the opposite electromigration fluxes for $\mathrm{Na}^{+}$and $\mathrm{Cl}^{-}$computed in the three-dimensional setup with the numerical model are shown in Figure 8a-c. The comparison between the analytical solution and the numerical model is reported as cross sectional map (Figure 7d), as well as vertical (Figure 7e) and horizontal (Figure 7f) profiles at the outlet. Such comparison shows a very good match between the analytical solution and the numerical multicomponent ionic transport simulation of a strong electrolyte in pure water in the fully 3-D domain.

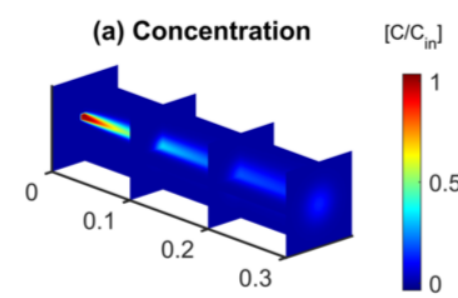

(d) Outlet Concentrations

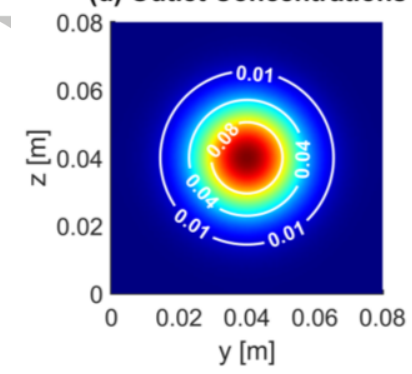

(b) $\mathrm{J}_{\mathrm{Na}}^{\mathrm{Mig}}$

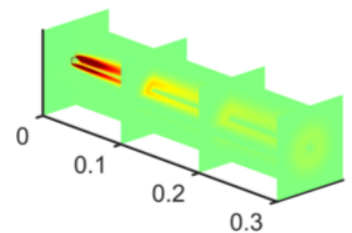

(e) Vertical profile

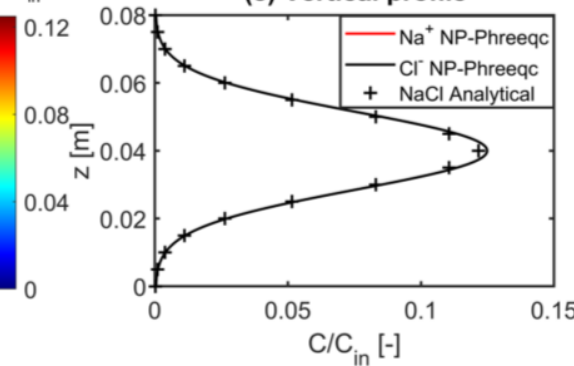

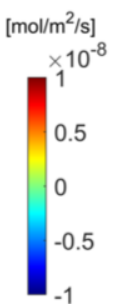
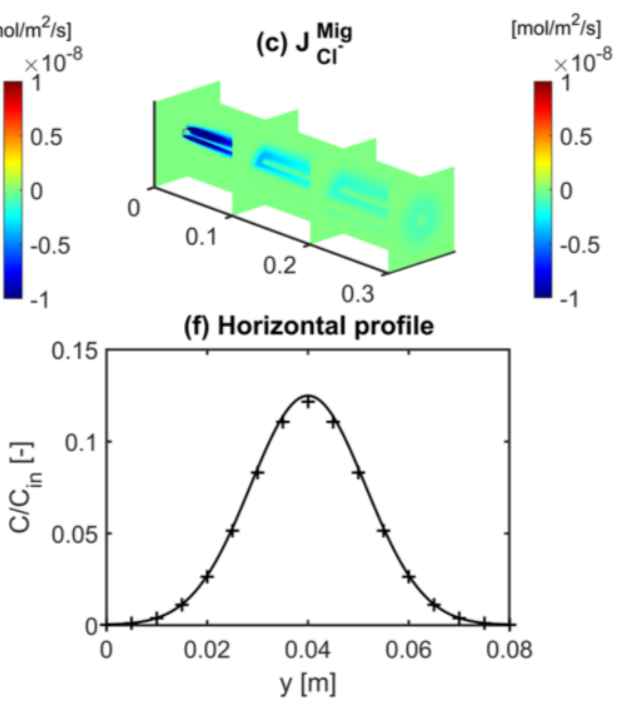

(f) Horizontal profile

Figure 8. Comparison of analytical solution and Nernst-Planck based ionic transport of $\mathrm{NaCl}$ in a 3-D setup. 3-D maps of the computed $\mathrm{Na}^{+}$plume (a) and transverse electromigration fluxes (considered positive in the direction from the core to the fringe of the plume) for the coupled $\mathrm{Na}^{+}$ and $\mathrm{Cl}^{-}$ions (b and c). Comparison between the analytical solution and the multicomponent ionic transport model at the outlet: 2-D visualization (d), vertical (e) and horizontal (f) profiles.

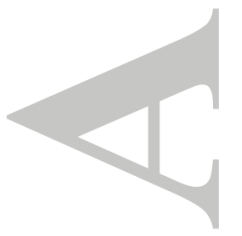




\subsubsection{3-D flow-through experiments}

Flow-through experiments are performed in this study to investigate the effects of multicomponent ionic dispersion during transport of charged species in fully three-dimensional setups. Details about the experimental setup are summarized in Table 4.

Table 4. Multicomponent ionic transport experiments in a 3-D flow-through setup.

\begin{tabular}{|c|c|c|c|}
\hline \multicolumn{2}{|l|}{ Experimental settings and hydraulic properties } & \multicolumn{2}{|r|}{ Value } \\
\hline \multicolumn{2}{|c|}{ Flow-through chamber inner dimensions $(L \times H \times W)[\mathrm{cm}]$} & \multicolumn{2}{|r|}{$30 \times 10 \times 7.7$} \\
\hline \multicolumn{2}{|l|}{ Source dimensions $\left(H_{s} \times W_{s}\right)[\mathrm{cm}]$} & \multicolumn{2}{|r|}{$1.54 \times 1.54$} \\
\hline \multicolumn{2}{|l|}{ Grain diameter of the porous matrix, $d[\mathrm{~mm}]$} & \multicolumn{2}{|r|}{$1.00-1.50$} \\
\hline \multicolumn{2}{|l|}{ Hydraulic conductivity, $K[\mathrm{~m} / \mathrm{s}]$} & \multicolumn{2}{|r|}{$\begin{array}{l}1.00-1.50 \\
1.27 \times 10^{-2}\end{array}$} \\
\hline \multicolumn{2}{|l|}{ Averäge horizontal flow velocity, $v[\mathrm{~m} /$ day $]$} & \multicolumn{2}{|r|}{0.5} \\
\hline \multicolumn{2}{|l|}{ Average porosity, $n[-]$} & \multicolumn{2}{|r|}{0.41} \\
\hline \multicolumn{4}{|l|}{ Transport experiments } \\
\hline $\begin{array}{l}\text { Plume } \\
\text { Background solution }\end{array}$ & $\begin{array}{c}\text { Experiment } 1 \\
\mathrm{MgCl}_{2} 0.30 \mathrm{mM} \\
\text { Milli-Q water }\end{array}$ & \multicolumn{2}{|c|}{$\begin{array}{c}\text { Experiment 2 } \\
\mathrm{MgCl}_{2} 0.30 \mathrm{mM} \\
\mathrm{NaBr} 1.70 \mathrm{mM}\end{array}$} \\
\hline Diffusion/dispersion coefficients at $\mathrm{T}=20^{\circ} \mathrm{C}$ & $D^{a q}\left[\mathrm{~m}^{2} / \mathrm{s}\right]$ & $D^{L}\left[\mathrm{~m}^{2} / \mathrm{s}\right]$ & $D^{T}\left[\mathrm{~m}^{2} / \mathrm{s}\right]$ \\
\hline $\mathrm{Na}^{+}$ & $1.20 \times 10^{-9}$ & $4.11 \times 10^{-9}$ & $1.06 \times 10^{-9}$ \\
\hline $\mathrm{Mg}^{2+}$ & $0.63 \times 10^{-9}$ & $3.88 \times 10^{-9}$ & $0.82 \times 10^{-9}$ \\
\hline $\mathrm{Cl}^{-}$ & $1.81 \times 10^{-9}$ & $4.36 \times 10^{-9}$ & $1.31 \times 10^{-9}$ \\
\hline $\mathrm{Br}^{-}$ & $1.86 \times 10^{-9}$ & $4.38 \times 10^{-9}$ & $1.34 \times 10^{-9}$ \\
\hline
\end{tabular}

Two experiments are performed in the 3-D system with continuous injection from an inlet port of a $\mathrm{MgCl}_{2}$ solution in Milli-Q water and in a background electrolyte solution of $\mathrm{NaBr}$, respectively. Samples are collected at the outlet of the setup where the steady-state plumes are mapped at high spatial resolution $(1.1 \mathrm{~cm}$ spacing) over a 2-D cross section. Measurements of the different ionic species are visualized as horizontal and vertical profiles through the central axis of the plume at the outlet cross section (Figure 9). A remarkable difference can be observed between the two flow-through experiments. A strong coupling between the plume cations and anions occurs when $\mathrm{MgCl}_{2}$ is released in Milli-Q water (Figure 9a). $\mathrm{Mg}^{2+}$ and $\mathrm{Cl}^{-}$undergo a similar displacement, despite the aqueous diffusion coefficient of chloride is approximately three times larger than the one of magnesium. In the experiments with release of a $\mathrm{MgCl}_{2}$ plume in the background electrolyte solution, instead, the plume cations and anions are less affected by the electrostatic coupling and they travel with hydrodynamic dispersion coefficients close to their liberated state. Therefore, their lateral displacement is different, with more spread horizontal and vertical profiles at the outlet for chloride due to its larger diffusion/dispersion coefficient. Such differences are remarkable despite the short length of the setup $(L=30 \mathrm{~cm})$. In fact, as shown in a recent comparison of solute transport in 2-D and 3-D systems (Ye et al., 2015a), in fully threedimensional domains the role of diffusion and compound-specific hydrodynamic dispersion is more relevant since these processes can act through the large material surface of threedimensional plumes. The simulations with the proposed multicomponent ionic transport model are able to capture the observed displacement of the ions in the 3-D flow-through setup. 

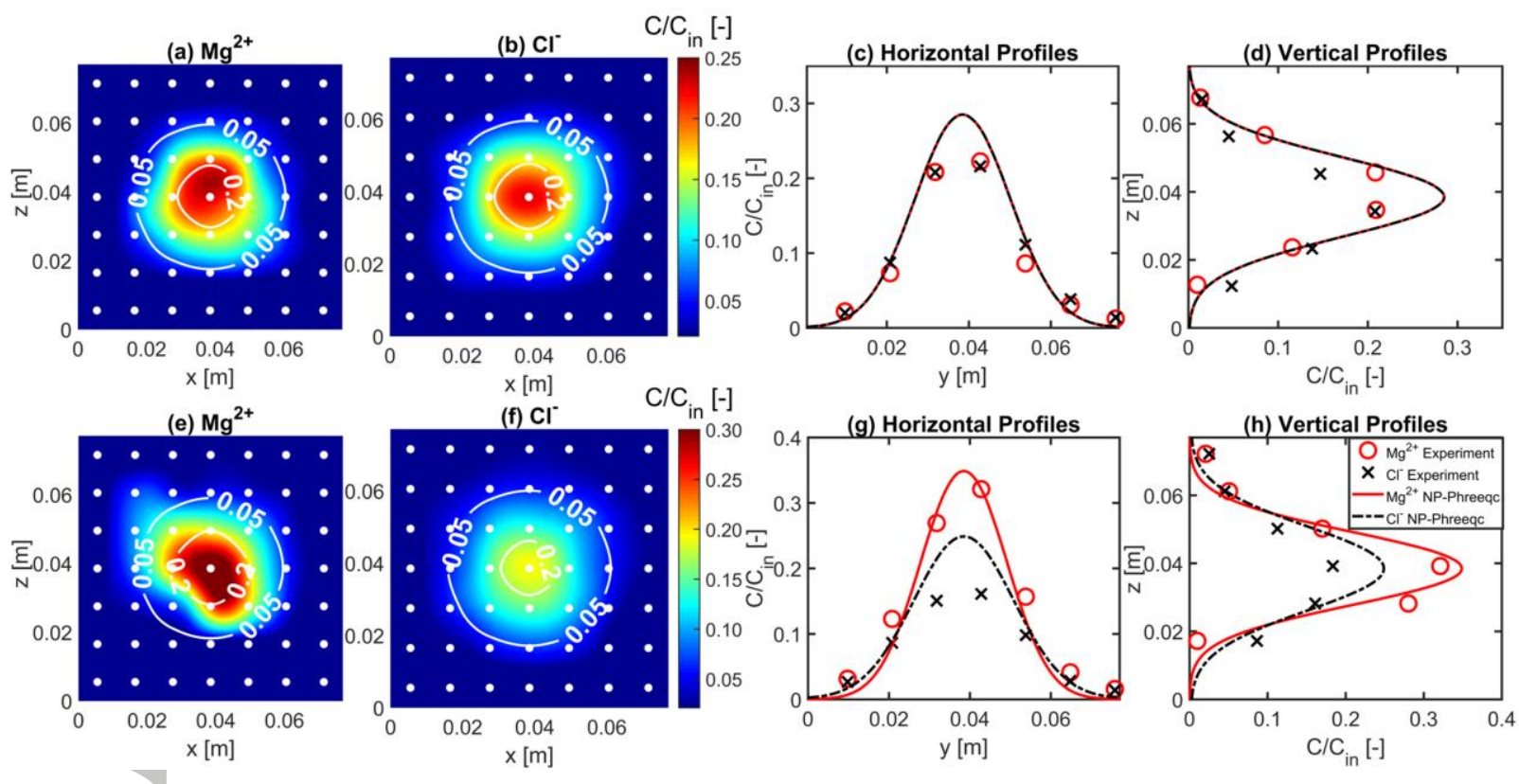

(g) Horizontal Profiles
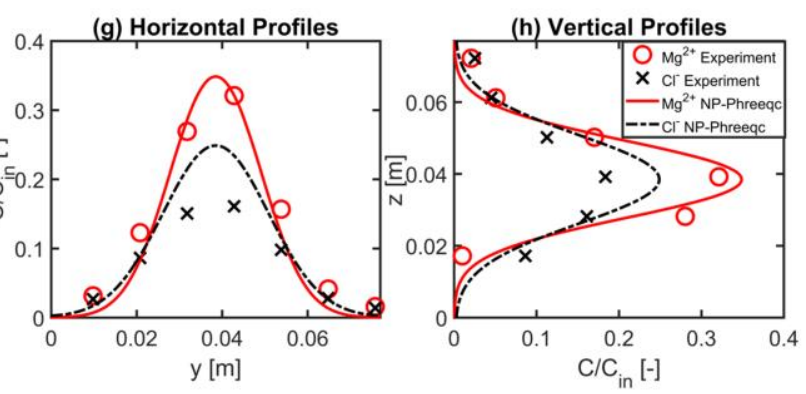

Figure 9. Measured and simulated horizontal and vertical profiles for the 3-D flow-through experiments: $\mathrm{MgCl}_{2}$ in Milli-Q water (a-d) and $\mathrm{MgCl}_{2}$ in $\mathrm{NaBr}$ solution (e-h).

Based on the 3-D simulations it is also possible to visualize the electromigration contribution to the Nernst-Planck hydrodynamic dispersive fluxes of the different ions during the two experiments. Figure 10 shows the results for the case of $\mathrm{MgCl}_{2}$ transport in Milli-Q water (panels a1 and b1) and in $\mathrm{NaBr}$ solutions (panels a2, b2, c2 and d2). In both cases the electromigration term has a positive contribution to the displacement of magnesium and a negative contribution to the displacement of chloride. However, the magnitude of the electromigration fluxes is quite different in the two cases. In fact, when $\mathrm{Mg}^{2+}$ and $\mathrm{Cl}^{-}$displace in Milli-Q water, their movement is coupled by the electromigration component which appears to be present in the whole domain at the fringe of the 3-D plume. When the same ions are transported in presence of higher concentration of background ions $\left(\mathrm{Na}^{+}\right.$and $\left.\mathrm{Br}^{-}\right)$, the latter counterbalance the charge differences and have a strong electromigration component whereas the coupling between $\mathrm{Mg}^{2+}$ and $\mathrm{Cl}^{-}$is almost suppressed and these plume ions can undergo a practically independent lateral displacement in the 3-D setup, resulting in the different profiles measured at the outlet of the flow-through chamber (Figure 9). 
$\mathrm{MgCl}$ in milliQ water

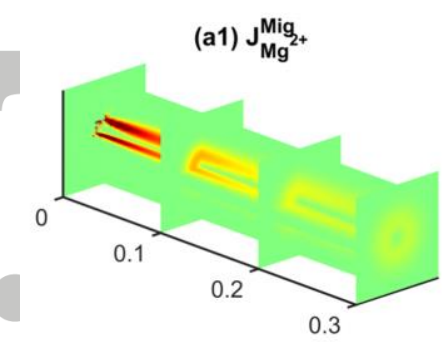

(b1) $\mathrm{J}_{\mathrm{Cl}}^{\mathrm{Mig}}$

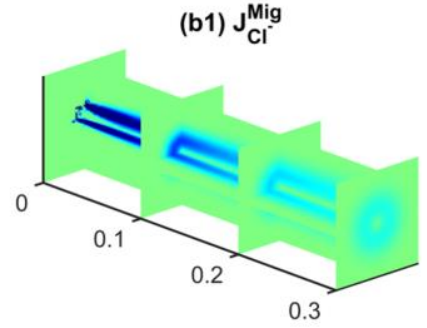

$\mathrm{MgCl}$ in $\mathrm{NaBr}$ solution
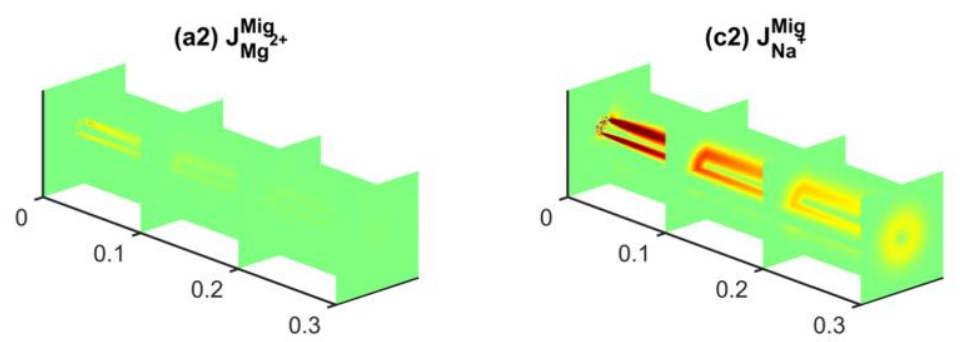

$\left[\mathrm{mol} / \mathrm{m}^{2} / \mathrm{s}\right]$

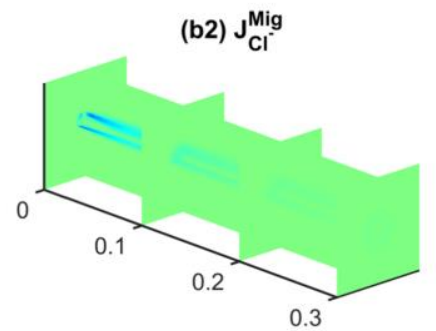

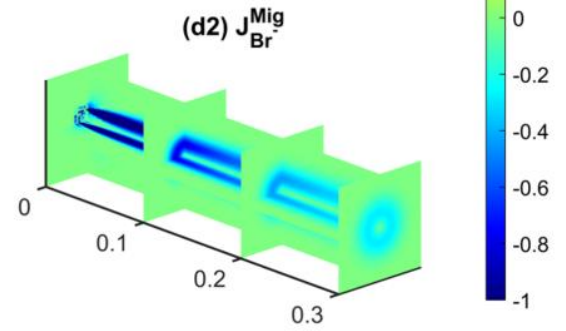

Figure 10. 3-D maps of the transverse electromigration fluxes computed for the two experiments: (i) transport of $\mathrm{MgCl}_{2}$ in Milli-Q water (a1 and b1) and (ii) transport of $\mathrm{MgCl}_{2}$ in $\mathrm{NaBr}$ solution (a2, b2, $\mathrm{c} 2$ and $\mathrm{d} 2$ ). The direction from the plume core to the plume fringe is considered positive for the computed fluxes.

\subsubsection{Multicomponent ionic transport and ion exchange}

As an example of multicomponent ionic displacement and reactive transport we consider a problem of ion exchange in a flow-through system under diffusion-dominated conditions, with a low seepage velocity $(v=0.024 \mathrm{~m} /$ day). The chemistry of the problem is the same as described in the example problem 11 of the PHREEQC-3 manual (Parkhurst \& Appelo, 2013). The $12 \mathrm{~cm}$ long exchanger column with $\mathrm{CEC}=0.0011 \mathrm{eq} / \mathrm{L}$ is initially in equilibrium with a solution of $\mathrm{NaNO}_{3}(1 \mathrm{mM})$ and $\mathrm{KNO}_{3}(0.2 \mathrm{mM})$ and is continuously flushed with a $\mathrm{CaCl}_{2}$ solution $(0.6$ $\mathrm{mM})$. As in the previous benchmarks, the species-specific aqueous diffusion coefficients are considered and the hydrodynamic dispersion coefficients are calculated according to Eqs. 6-7. The values of these parameters are reported in Table 5 together with the geometry and parameters of the column setup.

Table 5. Ion exchange in a column setup.

Setup geometry and parameters

Length of the column [cm]

Diameter of the column [cm]

Number of elements in 3-D NP-Phreeqc

Grain diameter of the porous matrix, $d[\mathrm{~mm}]$

Average flow velocity, $v$ [m/day]

Average porosity, $n[-]$

Cation Exchange Capacity, $C E C$ [eq/L]

0.0011

Displacing solution:

$\mathrm{CaCl}_{2}[\mathrm{mM}]$

Initial solution:

$\mathrm{NaNO}_{3}[\mathrm{mM}]$ 


\begin{tabular}{lccc}
\hline Diffusion/dispersion coefficients at $\mathbf{T}=\mathbf{2 0}^{\mathbf{0}} \mathbf{C}$ & $D^{a q}\left[\mathrm{~m}^{2} / \mathrm{s}\right]$ & $D^{L}\left[\mathrm{~m}^{2} / \mathrm{s}\right]$ & $D^{T}\left[\mathrm{~m}^{2} / \mathrm{s}\right]$ \\
\hline $\mathrm{Na}^{+}$ & $1.20 \times 10^{-9}$ & $0.63 \times 10^{-9}$ & $0.51 \times 10^{-9}$ \\
$\mathrm{~K}^{+}$ & $1.77 \times 10^{-9}$ & $0.86 \times 10^{-9}$ & $0.75 \times 10^{-9}$ \\
$\mathrm{Ca}^{2+}$ & $0.71 \times 10^{-9}$ & $0.43 \times 10^{-9}$ & $0.31 \times 10^{-9}$ \\
$\mathrm{NO}_{3}^{-}$ & $1.70 \times 10^{-9}$ & $0.84 \times 10^{-9}$ & $0.51 \times 10^{-9}$ \\
$\mathrm{Cl}^{-}$ & $1.81 \times 10^{-9}$ & $0.88 \times 10^{-9}$ & $0.77 \times 10^{-9}$ \\
\hline
\end{tabular}

The multicomponent ionic transport and ion exchange in the column are simulated as 1-D system using PHREEQC as well as a fully 3-D setup with the proposed COMSOL-PhreeqcRM coupling. In the latter case, the exchanger column was simulated assuming a diameter of $2 \mathrm{~cm}$ and using 2400 elements to discretize the three-dimensional domain. The 3-D NP-Phreeqc model solves Eq. 4 for the three-dimensional transport and the ion exchange problem. Figure 11a shows the breakthrough curves of the different ionic species at the outlet of the exchange column. The breakthrough profiles are determined by the exchange reactions and by multicomponent ionic transport. The equilibrium reactions between the dissolved ions and the exchanger determine the sequence of ions' breakthrough curves observed in the effluent. Among the cations, sodium is the one that exchanges more weakly and is the first to be eluted, followed by potassium and finally by calcium. The effects of charge interactions during transport of the different ions is particularly visible in the chloride breakthrough curve, which does not have the smooth shape expected for a charge neutral conservative tracer. In particular at later times, when chloride is the only dissolved anion, its profile is influenced by the charge interactions with the other species in solution. Such effect is particularly remarkable after $\sim 1.9 \mathrm{PVs}$ when the breakthrough of $\mathrm{Ca}^{2+}$ occurs and the concentration of $\mathrm{Cl}^{-}$increases to fulfill the charge balance. The 1-D PHREEQC and 3-D NP-Phreeqc simulations show a very good agreement, which validates the proposed Nernst-Planck based coupling for a fully three-dimensional transient reactive transport problem. Figure $11 \mathrm{~b}$ shows maps of the components of the longitudinal dispersive fluxes in the 3-D column after 1.5 PVs. The plots highlight the uphill dispersion of chloride that can be observed as a peak of concentration higher than the injected value. This uphill peak of chloride appears at the column outlet after flushing approximately two pore volumes. Such behavior is induced by the coupling of $\mathrm{Cl}^{-}$with the cations $\left(\mathrm{K}^{+}\right.$and $\left.\mathrm{Ca}^{2+}\right)$ which are released from the exchanger to the pore water. This can also be explained with the surprisingly higher magnitude (comparable with the purely dispersive flux component) of the electromigration flux, which, in fact, forces $\mathrm{Cl}^{-}$to establish this secondary concentration peak against its own concentration gradient in order to compensate the surplus of positive charge from the desorbed cations. 

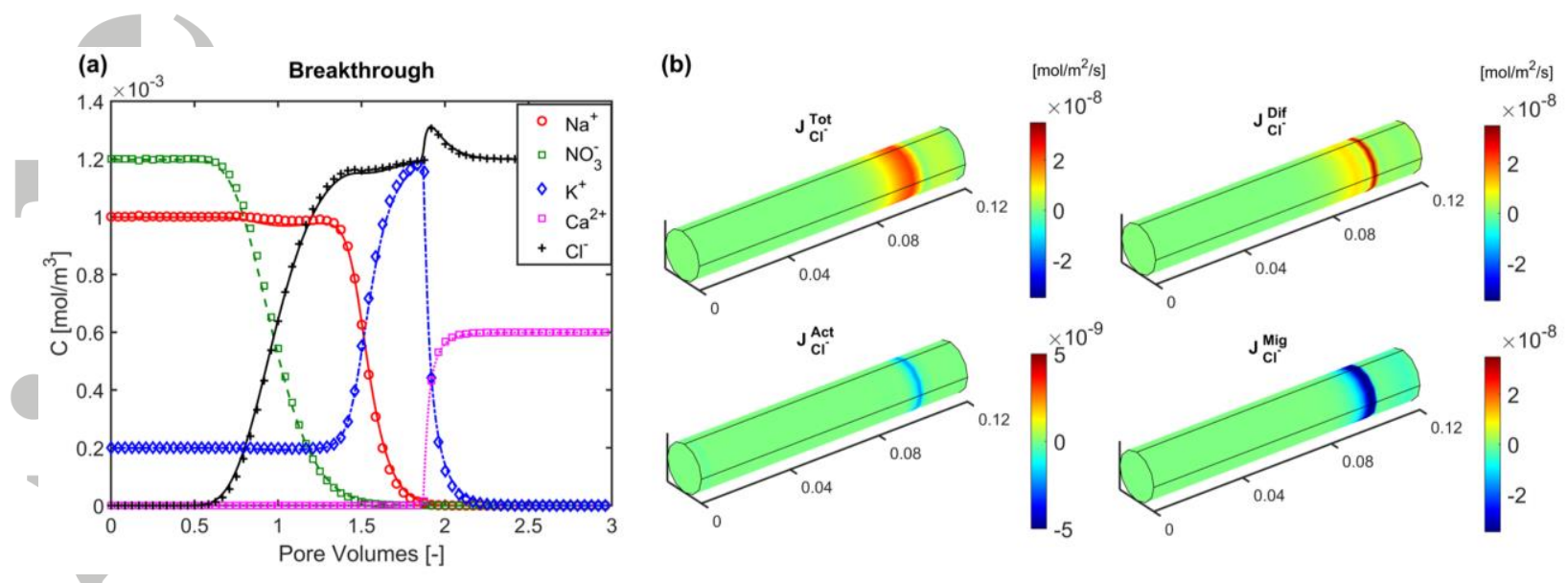

Figure 11. Comparison of breakthrough curves for 3-D simulation results with NP-Phreeqc (lines) and 1-D PHREEQC (symbols) for ion exchange and multicomponent ionic transport in a column setup (a). Maps of the longitudinal dispersive fluxes in the 3-D column after 1.5 pore volumes (b). The direction from high to low concentration values is considered positive for the computed fluxes.

\section{Conclusions}

In this paper we have proposed a Nernst-Planck based approach to describe conservative and reactive transport of charged species in saturated porous media. The approach was implemented as a multidimensional COMSOL-PhreeqcRM coupling. Key features of the proposed multicomponent ionic transport code are: (i) transport of all aqueous species (and not only of chemical components) with species-dependent coefficients; (ii) flexibility in the simulation of multidimensional domains, complex geometries, as well as heterogeneous media; (iii) wide range of geochemical reactions, including equilibrium and kinetic processes, ensured by the coupling with the geochemical code PHREEQC; (iv) calculation of all terms in NernstPlanck diffusive/dispersive fluxes including the contribution due to the gradients of activity coefficients that become important in concentrated solutions and in presence of strong variations of ionic strength. The code was validated against analytical solutions, numerical multicomponent ionic transport simulations carried out with PHREEQC in 1-D equivalent setups, and highresolution experimental datasets. Moreover, we performed experiments in a flow-through laboratory setup to investigate the effects of Coulombic interactions during fully threedimensional transport. The collected dataset allowed us to benchmark the proposed NP-Phreeqc model for 3-D multicomponent ionic transport.

The applications presented in this study have addressed simulation scenarios and experimental datasets focusing on multicomponent ionic transport. All the benchmark examples analyzed show the key importance of charge interactions on conservative and reactive solute transport. Electrostatic interactions in porous media are relevant for charged species transport not only in diffusive regimes but also under flow-through conditions. The outcomes of this investigation highlight the need of the proposed model to quantitatively describe Coulombic effects in flow-through porous media. The capability of the model to map individual components of Nernst-Planck diffusive dispersive fluxes and their spatial and temporal variability is an important feature providing key mechanistic insights to understand and correctly interpret 
measured concentrations of charged species in flow-through systems. Thus, NP-Phreeqc represents a unique tool to accurately simulate multicomponent ionic transport (from 1-D to 3-D) and to correctly interpret concentration measurements in both homogeneous and heterogeneous domains. The flexibility of the coupling offers the opportunity to extend the proposed multidimensional Nernst-Planck based approach to many relevant reactive transport processes in groundwater, including mineral precipitation and dissolution, displacement of radionuclides in flow-through systems, transport of tracer isotopes, mobilization of heavy metals and metalloids, propagation of acidic plumes, and degradation reactions (e.g., Kjøller et al., 2004; Maher et al., 2006; Molins et al., 2012; Prigiobbe et al., 2012; Glaus et al. 2014; Prigiobbe \& Bryant, 2014; Druhan et al., 2015; Haberer et al., 2015; Fakhreddine et al., 2016; McNeece \& Hesse, 2016 and 2017). Besides applications at the continuum scale, further developments are envisioned also at the pore scale where the proposed COMSOL-PhreeqcRM coupling could be extended to different physics and governing equations such as Stokes flow and multidimensional multicomponent ionic diffusion. This will allow exploring fundamental aspects of the coupling between fluid dynamics and mass transfer in porous media, Coulombic effects, solid-solution interactions and geochemical reactions. Finally, the flow-through perspective on multicomponent ionic transport in porous media and the multidimensional Nernst-Planck based approach introduced in this study could be extended to simulate engineering applications in which an electric field is applied on a porous medium to enhance and/or control transport of charged species. Examples of such applications include solute transport in concrete (Appelo, 2017), as well as electrokinetic for remediation of contaminated matrices (Reddy \& Cameselle, 2009) and for in-situ leaching and recovery of metals (Martens et al., 2018).

\section{Acknowledgments, Samples, and Data}

The experimental data for the 3-D multicomponent ionic transport experiments performed in this study are provided in the Supporting Information (Table S3). The document also provides tables (Tables S1 and S2) summarizing the data of previous experimental studies used in this work to benchmark the proposed approach for 2-D multicomponent ionic transport.

The authors thank Mikael Emil Olsson for assistance in performing the IC and ICP-MS measurements. M.R. acknowledges the support of the Sino-Danish Center for Education and Research, the German Research Foundation (grant RO4169/2-1) and the Villum Foundation (Block's Fellowship). Constructive comments from Dr. Christophe Tournassat and two anonymous reviewers helped improving the quality of the paper. 


\section{References}

Alt-Epping, P., C. Tournassat, P. Rasouli, C. Steefel, K. U. Mayer, A. Jenni, U. Mäder, S. Sengor, \& R. Fernandez (2015), Benchmark reactive transport simulations of a column experiment in compacted bentonite with multispecies diffusion and explicit treatment of electrostatic effects, Computat Geosci, 19(3), 535-550.

Appelo, C. A. J., \& D. Postma (2005), Geochemistry, groundwater and pollution, 649 pp., Balkema, Leiden, The Netherlands.

Appelo, C. A. J., \& P. Wersin (2007), Multicomponent diffusion modeling in clay systems with application to the diffusion of tritium, iodide, and sodium in opalinus clay, Environ. Sci. Technol., 41(14), 5002-5007.

Appelo, C. A. J., A. Vinsot, S. Mettler, \& S. Wechner (2008), Obtaining the porewater composition of a clay rock by modeling the in- and out-diffusion of anions and cations from an in-situ experiment, J. Contam. Hydrol., 101(1-4), 67-76.

Appelo, C. A. J. \& M. Rolle (2010). PHT3D: a reactive multicomponent transport model for saturated porous media, Ground Water, 48, 627-632.

Appelo, C. A. J. (2017). Solute transport solved with the Nernst-Planck equation for concrete pores with "free" water and a double layer, Cement and Concrete Research, 101, 102-113.

Bard, A. J., \& L. R. Faulkner (2001), Electrochemical Methods: Fundamentals and Applications, second ed., 833 pp., John Wiley, New York.

Barry, D. A., H. Prommer, C. T. Miller, P. Engesgaard, A. Brun \& C. Zheng (2002), Modelling the fate of oxidisable organic contaminants in groundwater, Adv. Water Resour., 25, 945-983.

Ben-Yaakov, S. (1972), Diffusion of sea water ions-I. Diffusion of sea water into a dilute solution, Geochim. Cosmochim. Acta, 36(12), 1395-1406.

Bethke, C. (1997), Modelling transport in reacting geochemical systems. CR Acad. Sci. IIa, 324, 513-528.

Bijeljic, B., \& M. J. Blunt (2007), Pore-scale modelling of transverse dispersion in porous media, Water Resour. Res., 43, W12S11, doi:10.1029/2006WR005700.

Boudreau, B. P., F. J. R. Meysman, \& J. J. Middelburg (2004), Multicomponent ionic diffusion in porewaters: Coulombic effects revisited, Earth Planet. Sci. Lett., 222(2), 653-666.

Cao, J., \& P. K. Kitanidis (1998), Pore-scale dilution of conservative solutes: An example, Water Resour. Res., 34(8), 1941-1949, doi:10.1029/98WR01468.

Carrera, J., X. Sanchez-Vila, I. Benet, A. Medina, G. Galarza, \& J. Guimera (1998), On matrix diffusion: formulations, solution methods and qualitative effects, Hydrogeol. J, 6(1), 178-190.

Charlton, S. R., \& D. L. Parkhurst (2011), Modules based on the geochemical model PHREEQC for use in scripting and programming languages. Comput. Geosci., 37, 1653-1663.

Chiogna, G., C. Eberhardt, P. Grathwohl, O. A. Cirpka, \& M. Rolle (2010), Evidence of compound-dependent hydrodynamic and mechanical transverse dispersion by multitracer laboratory experiments, Environ. Sci. Technol., 44(2), 688-693.

Chiogna, G., O. A. Cirpka, P. Grathwohl, \& M. Rolle (2011), Relevance of local compoundspecific transverse dispersion for conservative and reactive mixing in heterogeneous porous media, Water Resour. Res., 47(7), W07540. 
Cirpka, O. A., F. P. J. de Barros, G. Chiogna, M. Rolle, \& W. Nowak (2011), Stochastic fluxrelated analysis of transverse mixing in two-dimensional heterogeneous porous media, Water Resour. Res., 47, W06515, doi:10.1029/2010WR010279.

Cirpka, O. A., M. Rolle, G. Chiogna, F. P. J. de Barros, \& W. Nowak (2012), Stochastic evaluation of mixing-controlled steady-state plume lengths in two-dimensional heterogeneous domains, J. Contam. Hydrol., 138-139, 22-39.

Cirpka, O. A., G. Chiogna, M. Rolle, \& A. Bellin (2015), Transverse mixing in threedimensional nonstationary anisotropic heterogeneous porous media, Water Resour. Res., 51(1), 241-260.

Crevacore, E., T. Tosco, R. Sethi, G. Boccardo, \& D. Marchisio (2016), Recirculation zones induce non-Fickian transport in three-dimensional periodic porous media, Phys. Rev. E, 94, 053118.

Cussler, E. L. (2009), Diffusion: Mass Transfer in Fluid Systems, third ed., 631 pp., Cambridge University Press, Cambridge.

de Anna, P., J. Jimenez-Martinez, H. Tabuteau, R. Turuban, T. Le Borgne, M. Derrien, \& Y. Méheust (2014), Mixing and reaction kinetics in porous media: An experimental pore scale quantification, Environ. Sci. Technol., 48, 508-516.

Delgado, J. M. P. Q., \& J. F. R. Carvalho (2001), Lateral dispersion in liquid flowthrough packed beds at Pe< 1400, Transp. Porous Med., 44, 165-180.

Delgado, J. M. P. Q. (2006), A critical review of dispersion in packed beds, Heat Mass Transfer., 42(4), 279-310.

Domenico, P. A., \& Palciauskas V. (1982), Alternative boundaries in solid waste management, Ground Water, 20, 303-311.

Druhan, J. L., S. T. Braun, \& C. Huber (2015), Isotopic gradients across fluid-mineral boundaries. Rev., Mineral. Geochem., 80, 355-391.

Eckert, D., M. Rolle, \& O. A. Cirpka (2012), Numerical simulation of isotope fractionation in steady-state bioreactive transport controlled by transverse mixing, J. Contam. Hydrol., 140141(0), 95-106.

Fakhreddine, S., J. Lee, P. K. Kitanidis, S. Fendorf \& M. Rolle (2016), Imaging geochemical heterogeneities using inverse reactive transport modeling: An example relevant for characterizing arsenic mobilization and distribution. Adv. Water Resour., 88, 186-197.

Giambalvo, E. R., C. I. Steefel, A. T. Fisher, N. D. Rosenberg, \& C. G. Wheat (2002), Effect of fluid-sediment reaction on hydrothermal fluxes of major elements, eastern flank of the Juan de Fuca Ridge, Geochim. Cosmochim. Acta, 66(10), 1739-1757.

Glaus, M. A., M. Birgersson, O. Karnland, \& L. R. Van Loon (2013), Seeming steady-state uphill diffusion of $22 \mathrm{Na}+$ in compacted montmorillonite, Environ. Sci. Technol., 47, 1152211527.

Guedes de Carvalho J. R. F., \& J. M. P. Q. Delgado (2005), Overall map and correlation of dispersion data for flow through granular packed beds, Chem. Eng. Sci., 60, 365-75.

Gvirtzman, H., \& S. M. Gorelick (1991), Dispersion and advection in unsaturated porous media enhanced by anion exclusion, Nature, 352, 793-795.

Haberer, C. M., M. Rolle, O. A. Cirpka, \& P. Grathwohl (2012), Oxygen transfer in a fluctuating capillary fringe, Vadose Zone J., 11(3). 
Haberer, C. M., M. Muniruzzaman, P. Grathwohl, \& M. Rolle (2015), Diffusive/Dispersive and reactive fronts in porous media: Fe (II)-oxidation at the unsaturated/saturated interface, Vadose Zone J., doi:10.2136/vzj2014.07.0091.

He W., C. Beyer, J. H. Fleckenstein, E. Jang, O. Kolditz, D. Naumov \& T. Kalbacher (2015), A parallelization scheme to simulate reactive transport in the subsurface environment with OGS\#IPhreeqc 5.5.7-3.1.2. Geosci. Model Dev., 8, 3333-3348.

Hadley, P. W., \& C. Newell (2014), The new potential for understanding groundwater contaminant transport, Groundwater, 52(2), 174-186.

Hochstetler, D. L., M. Rolle, G. Chiogna, C. M. Haberer, P. Grathwohl, \& P. K. Kitanidis (2013), Effects of compound-specific transverse mixing on steady-state reactive plumes: Insights from pore-scale simulations and Darcy-scale experiments, Adv. Water Resour., 54, 1-10.

Jara D., J. R. de Dreuzy \& B. Cochepin (2017), TReacLab: An object-oriented implementation of non-intrusive splitting methods to couple independent transport and geochemical software, Comput. Geosci., 109, 281-294.

Kjøller, C., D. Postma, \& F. Larsen (2004), Groundwater acidification and the mobilization of trace metals in a sandy aquifer, Environ. Sci. Technol., 38(10), 2829-2835.

Kolditz, O,, S. Bauer, L. Bilke, N. Böttcher, J. O. Delfs, T. Fischer, \& B. Zehner (2012), OpenGeoSys: An open-source initiative for numerical simulation of thermo-hydromechanical/chemical (THM/C) processes in porous media, Environ. Earth Sci., 67, 589-599.

Korrani, A. K. N., K. Sepehrnoori \& M. Delshad (2015), Coupling IPhreeqc with UTCHEM to model reactive flow and transport, Comput. Geosci., 82, 152-169.

LaBolle, E. M., \& G. E. Fogg (2001), Role of molecular diffusion in contaminant migration and recovery in an alluvial aquifer system, Transp. Porous Med., 42(1-2), 155-179.

Lasaga, A. C. (1979), The treatment of multi-component diffusion and ion pairs in diagenetic fluxes, Am. J. Sci., 279(3), 324-346.

Lasaga, A. C. (1998), Kinetic Theory in the Earth Sciences, 811 pp., Princeton University Press, Princeton, N.J.

Leij, F. J., T. H. Skaggs, \& M. T. Van Genuchten (1991), Analytical solutions for solute transport in three-dimensional semi-infinite porous media, Water Resour. Res., 27(10), 27192733.

Li, L., C. I. Steefel, \& L. Yang (2008), Scale dependence of mineral dissolution rates within single pores and fractures, Geochim. Cosmochim. Acta, 72(2), 360-377.

$\mathrm{Li}$, L., et al. (2017), Expanding the role of reactive transport models in critical zone processes, Earth Sci. Rev., 165, 280-301.

Lide, D.R. (2005), Handbook of Chemistry and Physics, $86^{\text {th }}$ Edition, 2544 pp.

Liu, C. X. (2007), An ion diffusion model in semi-permeable clay materials, Environ. Sci.

Technol., 41(15), 5403-5409.

Liu, C. X., J. Shang, \& J. M. Zachara (2011), Multispecies diffusion models: A study of uranyl species diffusion, Water Resour. Res., 47, W12514, doi:10.1029/2011WR010575.

Maher, K., C. I. Steefel, D. J. DePaolo, \& B. E. Viani (2006), The mineral dissolution rate conundrum: Insights from reactive transport modeling of $U$ isotopes and pore fluid chemistry in marine sediments, Geochim. Cosmochim. Acta, 70(2), 337-363. 
Maier, U., \& P. Grathwohl (2006), Numerical experiments and field results on the size of steady state plumes, J. Contam. Hydrol., 85, 33-52.

Martens, E., H. Prommer, D. Xianwen, M. Z. Wu, J. Sun, P. Breuer, \& A. Fourie (2018), Feasibility of electrokinetic in situ leaching of gold, Hydrometallurgy, 175, 70-78.

Masi M., A. Ceccarini, \& R. Iannelli (2017). Multispecies reactive transport modelling of electrokinetic remediation of harbour sediments, J. Hazard. Mater., 326, 187-196.

Mayer, K. U., E. O. Frind \& D. W. Blowes (2002), Multicomponent reactive transport modeling in variably saturated porous media using a generalized formulation for kinetically controlled reactions, Water Resour. Res., 38, 1301-1321.

McNeece, C. J., \& M. A. Hesse (2016), Reactive transport of aqueous protons in porous media, Adv. Water Resour., 97, 314-325.

McNeece C. J., \& M. A. Hesse (2017), Challenges in coupling acidity and salinity transport, Environ. Sci. Technol., 51 (20), 11799-11808.

Molins, S., \& U. K. Mayer (2007), Coupling between geochemical reactions and multicomponent gas and solute transport in unsaturated media: A reactive transport modeling study, Water Resour. Res., 43, W05435, 1-16.

Molins, S., D. Trebotich, C. I. Steefel, \& C. P. Shen (2012), An investigation of the effect of pore scale flow on average geochemical reaction rates using direct numerical simulation, Water Resour. Res., 48, W03527. http://dx.doi.org/10.1029/2011WR011404.

Muniruzzaman, M., C. M. Haberer, P. Grathwohl, \& M. Rolle (2014), Multicomponent ionic dispersion during transport of electrolytes in heterogeneous porous media: Experiments and model-based interpretation, Geochim. Cosmochim. Acta, 141(0), 656-669.

Muniruzzaman, M., \& M. Rolle (2015), Impact of multicomponent ionic transport on $\mathrm{pH}$ fronts propagation in saturated porous media, Water Resour. Res., 51(8), 6739-6755.

Muniruzzaman, M., \& M. Rolle (2016), Modeling multicomponent ionic transport in groundwater with IPhreeqc coupling: Electrostatic interactions and geochemical reactions in homogeneous and heterogeneous domains, Adv. Water Resour., 98, 1-15.

Muniruzzaman, M. \& M. Rolle (2017). Experimental investigation of the impact of compoundspecific dispersion and electrostatic interactions on transient transport and solute breakthrough. Water Resour. Res., 53(2), 1189-1209.

Nardi, A., A. Idiart, P. Trinchero, L. M. de Vries \& J. Molinero (2014), Interface COMSOLPHREEQC (iCP), an efficient numerical framework for the solution of coupled multiphysics and geochemistry. Comput. Geosci., 69, 10-21.

Parkhurst, D. L., K. L. Kipp, P. Engesgaard \& S. R. Charlton (2004), PHAST - A program for simulating ground-water flow, solute transport, and multicomponent geochemical reactions, US Geological survey techniques and methods 6, A8.

Parkhurst, D. L., \& C. A. J. Appelo (2013), Description of input and examples for PHREEQC version 3- A computer program for speciation, batch-reaction, one dimensional transport, and inverse geochemical calculations. U.S. Geological Survey Techniques and Methods; 6-A43:497.

Parkhurst, D. L., \& L. Wissmeier (2015), PhreeqcRM: A reaction module for transport simulators based on the geochemical model PHREEQC, Adv. Water Resour. 83, 176-189. 
Post, V., \& H. Prommer (2007), Multicomponent reactive transport simulation of the Elder problem: Effects of chemical reactions on salt plume development, Water Resour. Res., 43, W10404.

Prigiobbe, V., M. A. Hesse \& S. L. Bryant (2012), Fast strontium transport induced by hydrodynamic dispersion and pH-dependent sorption, Geophys. Res. Lett., 39, L18401.

Prigiobbe, V., \& S. L. Bryant (2014), pH-dependent transport of metal cations in porous media, Environ. Sci. Technol., 48(7), 3752-3759.

Probstein, R. F. (1989), Physicochemical hydrodynamics: An introduction, 337 pp., Butterworth Publishers, Stoneham.

Prommer, H., D. A. Barry \& C. Zheng (2003), MODFLOW/MT3DMS-based reactive multicomponent transport modeling, Ground Water, 41, 247-257.

Rasouli, P., C. I. Steefel, K. U. Mayer \& M. Rolle (2015). Benchmarks for multicomponent diffusion and electrochemical migration, Computat Geosci, 19(3), 523-533.

Reddy, K. R., \& C. Cameselle (2009), Electrochemical Remediation Technologies for Polluted Soils, Sediments and Groundwater, 732 pp. John Wiley \& Sons, Hoboken, NJ.

Rolle, M., C. Eberhardt, G. Chiogna, O. A. Cirpka, \& P. Grathwohl (2009), Enhancement of dilution and transverse reactive mixing in porous media: Experiments and model-based interpretation, J. Contam. Hydrol., 110(3-4), 130-142.

Rolle, M., D. L. Hochstetler, G. Chiogna, P. K. Kitanidis, \& P. Grathwohl (2012), Experimental investigation and pore-scale modeling interpretation of compound-specific transverse dispersion in porous media, Transp. Porous Med., 93(3), 347-362.

Rolle, M., M. Muniruzzaman, C. M. Haberer, \& P. Grathwohl (2013a), Coulombic effects in advection-dominated transport of electrolytes in porous media: Multicomponent ionic dispersion, Geochim. Cosmochim. Acta, 120(0), 195-205.

Rolle, M., G. Chiogna, D. L. Hochstetler, \& P. K. Kitanidis (2013b), On the importance of diffusion and compound-specific mixing for groundwater transport: An investigation from pore to field scale, J. Contam. Hydrol., 153(0), 51-68.

Rolle, M., \& P. K. Kitanidis (2014), Effects of compound-specific dilution on transient transport and solute breakthrough: A pore-scale analysis, Adv. Water Resour., 71, 186-199.

Scheven, U. M. (2013), Pore-scale mixing and transverse dispersivity of randomly packed monodisperse spheres, Phys. Rev. E, 110(21), 214504. doi: 10.1103/PhysRevLett.110.214504.

Steefel, C. I., \& A. C. Lasaga (1994), A coupled model for transport of multiple chemical species and kinetic precipitation dissolution reactions with application to reactive flow in single-phase hydrothermal systems. Am. Journal Sci., 294,529-592.

Steefel, C. I., \& K. Maher (2009), Fluid-rock interaction: A reactive transport approach, Rev. Mineral. Geochem., 70, 485-532.

Steefel, C. I., C. A. J. Appelo, B. Arora, D. Jacques, T. Kalbacher, O. Kolditz, \& G. T. Yeh (2015) Reactive transport codes for subsurface environmental simulation, Computat Geosci, 19, 445-78.

Tartakovsky, A. M., G. D. Tartakovsky, \& T. D. Scheibe (2009), Effects of incomplete mixing on multicomponent reactive transport, Adv. Water Resour., 32(11), 1674-1679, doi:10.1016/j.advwatres.2009.08.012. 
Thullner, M., P. Van Cappellen, \& P. Regnier (2005), Modeling the impact of microbial activity on redox dynamics in porous media, Geochim. Cosmochim. Acta, 69(21), 5005-5019.

Tournassat, C., \& C. A. J. Appelo (2011), Modelling approaches for anion exclusion in compacted Na-bentonite, Geochim. Cosmochim. Acta, 75, 3698-3710.

Tournassat, C., \& C. I. Steefel (2015), Ionic transport in nano-porous clays with consideration of electrostatic effects, Rev. Mineral. Geochem., 80, 287-329.

Van Breukelen, B. M., \& M. Rolle (2012), Transverse hydrodynamic effects on isotope signals in groundwater chlorinated solvents' plumes, Environ. Sci. Technol., 46, 7700-7708.

van Genuchten, M. T., F. J. Leij, T. H. Skaggs, N. Toride, S. A. Bradford, \& E. M. Pontedeiro (2013), Exact analytical solutions for contaminant transport in rivers 1 . The equilibrium advection-dispersion equation, J. Hydrol. Hydromech., 61(2), 146-160.

Werth, C. J., O. A. Cirpka, \& P. Grathwohl (2006), Enhanced mixing and reaction through flow focusing in heterogeneous porous media, Water Resour. Res., 42, W12414, doi:10.1029/2005WR004511.

Wissmeier, L., \& D. A. Barry (2011), Simulation tool for variably saturated flow with comprehensive geochemical reactions in two- and three-dimensional domains, Environ. Model. Softw., 26, 210-218.

Xu, T., \& K. Pruess (2001), Modeling multiphase non-isothermal fluid flow and reactive geochemical transport in variably saturated fractured rocks 1: Methodology, Am. J. Sci., 301, 1633.

Ye, Y., G. Chiogna, O. Cirpka, P. Grathwohl, \& M. Rolle (2015a), Experimental investigation of compound-specific dilution of solute plumes in saturated porous media: 2-D vs. 3-D flowthrough systems, J. Contam. Hydrol., 172, 33-47.

Ye, Y., G. Chiogna, O. A. Cirpka, P. Grathwohl, \& M. Rolle (2015b), Enhancement of plume dilution in two-dimensional and three-dimensional porous media by flow focusing in highpermeability inclusions, Water Resour. Res., 51(7), 5582-5602.

Ye, Y., G. Chiogna, O. A. Cirpka, P. Grathwohl, \& M. Rolle (2015c), Experimental evidence of helical flow in porous media, Phys. Rev. Lett., 115(19), 194502.

Yoon, H., A. J. Valocchi, C. J. Werth \& T. Dewers (2012), Pore-scale simulation of mixinginduced calcium carbonate precipitation and dissolution in a microfluidic pore network. Water Resour. Res., 48(2).

Zhang, C. Y., K. Dehoff, N. Hess, M. Oostrom, T. W. Wietsma, A. J. Valocchi, B. W. Fouke, \& C. J. Werth (2010), Pore-scale study of transverse mixing induced $\mathrm{CaCO}_{3}$ precipitation and permeability reduction in a model subsurface sedimentary system, Environ. Sci. Technol., 44(20), 7833-7838.

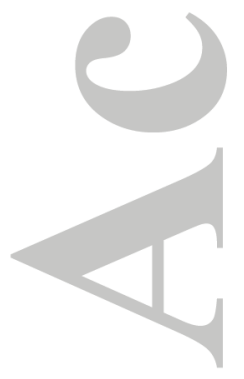

